Article

\title{
Financial Variables, Market Transactions, and Expectations as Functions of Risk
}

\author{
Victor Olkhov \\ TVEL, Moscow 115409, Russia; victor.olkhov@gmail.com
}

Received: 27 August 2019; Accepted: 29 October 2019; Published: 4 November 2019

\begin{abstract}
This paper develops methods and a framework of financial market theory. We model financial markets as a system of agents which perform market transactions with other agents under the action of numerous expectations. Agents' expectations are formed of economic and financial variables, market transactions, the expectations of other agents, and other factors that impact financial markets. We use the risk ratings of agents as their coordinates and approximate a description of financial variables, market transactions, and expectations of numerous separate agents by density functions of aggregated agents in the economic domain. The motion of separate agents in the economic domain due to a change of agents' risk rating produces collective financial flows of variables, transactions, and expectations. We derive equations on collective financial variables, market transactions, expectations, and their flows in the economic domain. These flows define the evolution of financial markets. As an example, we present a simple model with linear dependence between disturbances of volume and the cost of transactions on one hand, and disturbances of expectations that determine transactions on the other hand. Our model describes harmonique oscillations of these disturbances with numerous frequencies and allows an explicit form for fluctuations of price and return to be derived. These relations show a direct dependence between price, return, and volume perturbations.
\end{abstract}

Keywords: financial markets; risk ratings; financial flows; density functions

JEL Classification: C00; E320; E440; G00; G120

\section{Introduction}

In recent decades, Econophysics has produced many studies for modeling financial markets and the statistical properties of price and return fluctuation: Mantegna and Stanley (2000), Gabaix et al. (2003), Stanley (2003), and Borland and Bouchaud (2005). The variety of financial instruments causes diversity of their response to shocks and requires the usage of different models and approximations (Roehner 2010; Chakraborti et al. 2011; Schinckus 2012; Gontis et al. 2016; Guedesa et al. 2018; Dhesi et al. 2019). The modeling statistical properties of financial markets require methods that describe general financial and economic relations of market trading and price evolution.

At present, financial market and macro finance modeling heavily rely on methods of general equilibrium theory (GE) (Arrow and Debreu 1954; Arrow 1974; Kydland and Prescott 1990; Starr 2011) and DSGE (Fernández-Villaverde 2010; Komunjer and Ng 2011; Del Negro et al. 2013; Farmer 2017). Existing flaws and weaknesses of GE and DSGE may bring unjustified decisions and excess shocks to unsteady financial processes. Numerous papers have discussed the pros and cons of GE (Hazlitt 1959; Morgenstern 1972; Ackerman 1999; Stiglitz 2017). A special issue of Oxford Review of Economic Policy on "Rebuilding macroeconomic theory" discusses: "What new ideas are needed? What needs to be thrown away? What might a new benchmark model look like? Will there be a 'paradigm shift'?" (Vines and Wills 2018a, 2018b). 
Financial markets and their dynamics are very complex and their description requires different methods and approximations. In this paper, we develop methods, models, and equations, which treat financial variables, market transactions, and expectations as functions of risk. We propose that such an approach to financial modeling may improve the understanding and description of real financial markets.

This paper will treat macro finance as a system of agents with economic and financial variables. We regard economic agents as simple units that define economic and financial relations of the macro financial system. Agent-based models (ABM) have been studied for decades, and we mention (Poggio et al. 1999; Tesfatsion and Judd 2005; Chakraborti et al. 2011; Janžek and Ziherl 2013; Haldane and Turrell 2018) and the references therein as only a small fraction of these studies. We note households, banks, industry companies, and all those who establish macroeconomic and financial systems as economic agents. Aggregations of economic and financial variables of agents define macro financial variables and market transactions between agents define the evolution of the macro financial system. Participants of financial markets-agents-are engaged in numerous market transactions with other agents. Agents perform their transactions under different expectations. Agents form their expectations on the basis of economic or financial variables, transactions, expectations of other agents, policy, technology or regulatory changes, and so on. Market transactions of agents are always performed under risks. Moreover, the financial activity of agents creates risks and no economic or financial development is possible without risks. We use risk ratings of agents as their coordinates and show that the change of an agent's risk rating due to any reason causes risk collective flows of financial variables, transactions, and expectations. These flows induce continuous changes of economic and financial variables like investment and credits, or market supply and demand in particular. The permanent evolution of market supply and demand due to risk flows makes the concept of equilibrium states questionable. In this study, we develop methods, tools, and equations that describe relations between financial variables, transactions, and expectations and their flows. A general approach description of financial variables, market transactions, and expectations allows model price and return fluctuations to act as the internal property of financial markets.

The rest of the paper unfolds as follows. In Section 2, we discuss assumptions, and introduce the economic domain, financial variables and their flows, and the main equations. In Section 3, we discuss financial transactions as functions of risk. In Section 4, we introduce expectations as functions of risk. To show the advantages of our approach, in Section 5, we apply our methods to model asset price and return fluctuations. The conclusion is presented in Section 6.

\section{Assumptions, Variables, and Equations}

The main assumptions, starting with the properties of financial variables, will now be discussed. Agents have different variables like credits and debts, assets and investment, and supply and demand. Some variables are additive and some are non-additive. For example, the sum of investment or credits of a group of agents (without doubling) defines the investment and credits of the entire group. Ratios of additive variables define non-additive variables like prices or financial ratios. Inflation and indexes are determined as the ratio of prices in different moments of time and are also non-additive. Therefore, an agent's additive financial or economic variables describe all economic and financial variables. The aggregation of agents' additive variables defines the macro variables. For example, the sum of an agent's credits (without doubling) determines the macroeconomic credits, the sum of an agent's investment defines the investment of all the economics, etc. Therefore, agents' additive variables are key factors that define the macro financial variables and their evolution.

Some additive variables are involved in market transactions between agents. Each transaction implies that the seller transfers a certain number of additive variables, like commodities, assets, service, investment, and credits, to the buyer. Transactions between agents are only factors that impact the change of additive variables. Changes of market regulation, political, technological or climate impacts on markets only have results after certain transactions are performed. The available information for 
the value and volume of the performed transactions and prices of the transactions may impact the change of variables for all agents. Therefore, a description of transactions between agents plays a key role for modeling all agents and financial variables.

All agents perform transactions under different expectations. Agents' expectations determine the volume and value of transactions, choice of commodities, amount of credits, and directing investment. Agents' expectations as drivers of transactions are responsible for financial activity and hence impact the evolution of macro variables. Agents form their expectations as forecasts of variables, expectations of other agents, market regulatory trends, technology, climate, and other changes. Therefore, expectations transfer the impact of endogenous and exogenous factors on the performance of transactions between agents and hence on the evolution of financial variables.

Relations between financial variables, transactions, and expectations establish a core problem for macro modeling. In this paper, we present methods and tools that describe the evolution of variables, transactions, and expectations under different approximations. We use bold italic font to denote vectors and italic font for scalars. Three main issues determine our approach:

- We use risk ratings of agents as their coordinates;

- We describe variables, transactions and expectations as functions of risk;

- We describe collective flows of financial variables, transactions, and expectations and their impact on financial markets.

These issues will now be discussed in detail.

\subsection{Risk Ratings of Agents as Their Coordinates}

We propose risk ratings of economic agents as their coordinates in economic space. A description of agents in economic space establishes the basis for modeling financial variables, market transactions between agents, and expectations as functions of time and coordinates in economic space. This magnifies the modeling capacity and uncovers the internal complexity of macro financial evolution. We introduce a description of economic agents in economic space (Olkhov 2016a, 2016b, 2017a, 2017b, 2017c, 2018a, $2018 \mathrm{~b}, 2019)$ and present different benefits and problems of such approach. Agent-based models in macro finance are well-known and numerous papers have presented various descriptions of financial markets, decision-making, etc. (Poggio et al. 1999; Tesfatsion and Judd 2005; Chakraborti et al. 2011; Janžek and Ziherl 2013; Haldane and Turrell 2018). However, we do not know of any studies that have used risk ratings as agents' coordinates similar to our model of economic space. For decades, international rating agencies such as S\&P, Moody's, and Fitch (Metz and Cantor 2007; S\&P 2014; Fitch Ratings 2018) have provided risk assessments for major banks, corporations, securities, etc., and delivered distributions of the biggest banks by their risk ratings (Moody's Investors Service 2018; South and Gurwitz 2018). These assessments determine the investment expectations of the biggest hedge funds, investors, traders, etc. According to current risk assessment methodologies (Altman et al. 2010; Moody's Investors Service 2010; S\&P 2016; Fitch Ratings 2018), risk ratings take values of risk grades like $A A A, A A, B B, C$, etc. Different rating agencies use different risk assessment methodologies and risk grade notions differ slightly.

Risk grades $A A A, A A, B B, C$ can be treated as points $x_{1}, \ldots x_{N}$ of space that we call economic space. The risk assessment methodology uses available economic statistics and determines the number $N$ of risk points. We propose that economic statistics and econometrics provide sufficient data to assess risk ratings for all economic agents and for all risks that may hit macroeconomic or macro financial evolution and growth. Additionally, we assume that rating agencies assess risk ratings for all agents, including large corporations and banks, for small companies and firms, and even households. Now we assume that the risk assessment methodologies define continuous spectrum of the risk grades in the space $R$. The risk methodology may always take the continuous risk grades as interval $[0,1]$ with point 0 as most secure and 1 as most risky grade. A lot of different risks may disturb the macroeconomic processes (McNeil et al. 2005). The assessment of single risk, like credit risk, distributes 
agents over interval [0,1] of 1-dimensional space $R$. The assessment of two or three risks, like credit, exchange rate, and liquidity risk for example, distributes economic agents over unit square or cube. For the given configuration of $n$ macro risks, assessment of the agents' risk rating distributes agents by risk coordinates $x=\left(x_{1}, \ldots x_{n}\right)$ in economic domain of $n$-dimensional space $R^{n}$. Distribution of economic agents by risk coordinates $x=\left(x_{1}, \ldots x_{n}\right)$ in economic domain (1) implies that all agent's economic and financial variables are also distributed in (1). Aggregation of the similar variables of agents with coordinates near point $x=\left(x_{1}, \ldots x_{n}\right)$ of (1) defines the collective variables as a function of $x$. Aggregations of the similar transactions between agents with coordinates $x$ and $y$ determine the collective transactions as a function of $x$ and $y$ in (1). This helps describe the financial variables, transactions, and expectations, by partial differential equations in economic domain.

$$
0 \leq x_{i} \leq 1, i=1, \ldots n
$$

\subsection{Variables, Transactions, and Expectations as Functions of Risk}

A description of financial variables, transactions, and expectations of separate agents of all economics is too complex and does not help model the evolution of macro variables. Up to now, macro financial modelling has used aggregations of variables of all agents as functions of time. For example, the sum of investment of all agents defines macro investment as a function of time. We claim that such an approach hides and loses too much information about the properties of financial macro variables and that this may be the origin of numerous failures of GE and DSGE. We use the distribution of agents in the economic domain (1) as the tool for a description of collective financial variables, transactions, and expectations as functions of risk. Such approximation is much rougher then description relations between variables, transactions, and expectations of separate agents, but much more detailed than descriptions as only functions of time. Let us chose economic space scale $d$ and time scale $\Delta$. Additionally let us collect variables, transactions, or expectations of agents with risk coordinates inside a small volume $d V=d^{n}$ near point $x$ of (1) and then average them during a certain time term $\Delta$. It can be assumed that the scale $d<<1$, but many economic agents have risk coordinates inside $d V$ near point $x$. It can also be assumed that time $\Delta$ is too small to compare with the time scale of the problem under consideration, but many transactions are performed during $\Delta$. For example, we can estimate the number of agents in economics with a population of $10^{8}-10^{9}$ as $10^{8}-10^{9}$. Therefore, the space scale $d \sim 10^{-2}$ in a two-dimensional domain (1) defines the volume $d V \sim$ $10^{-4}$, with around $10^{4}-10^{5}$ agents inside it. Time scale $\Delta=1$ week is too small to compare it with the time term of one quarter or year. It can be assumed that agents perform one transaction per second, and there are thus about $6 \times 10^{5}$ transactions per $\Delta=1$ week. Therefore, aggregation by scales $d \sim 10^{-2}$ and averaging by $\Delta=1$ week may approximate economic processes for a time term of one quarter, year, or more. As an example, we can consider the credits provided by agents inside $d V$ near point $x$ and average them during $\Delta=1$ week. Let us consider that $C(t, x)$ equals the sum of credits provided by agents in volume $d V$ and averaged during time $\Delta$. Function $C(t, x)$ represents the density of credits provided by agents from point $x$ at moment $t$. Indeed, the integral of $C(t, x)$ by $d x$ over economic domain equals the total credits provided by all agents in economics at moment $t$. Averaging over time $\Delta$ reduces high-frequency fluctuations of the collected credits and makes this variable smooth. The introduction of scale $d$ and scale $\Delta$ reduces the accuracy of the model approximation. If one choses scale $d=1$, then volume $d V$ will be equal to the economic domain (1) and the sum of credits provided by agents inside (1) equals all credits provided in macroeconomics. Similar definitions allow collective transactions between two points $x$ and $y$ on (1) to be introduced as density functions of two risk coordinates and density functions of expectations as functions of $x$. Therefore, the introduction of scales $d<<1$ establishes approximation that is intermediate between a precise description of separate economic agents and a too rough approximation based on the aggregation of variables of all agents in the economy. Below, we define density functions of financial variables, transactions, and expectations. 
It is obvious that one may aggregate agents and their variables, transactions, and expectations in domain (1) by various economic or financial groups with sections for different industry sectors, wealth, gender, age, or other economic or financial specification. Macro models based on the aggregation of agents by various groups in domain (1) may model relations between banks and industry sectors or describe the influence of any specifications that define grouping agents. Such models may use different sets of risks and different risk measures for different groups of agents. For example, risk assessments may differ for financial and industry sectors, or for different wealth. Any specific grouping and usage of different sets of risks and risk measures introduce additional complexity to the model. In the current paper, we describe the simplest tools and framework without any additional specifications.

The most important factor that impacts the evolution of density functions of variables, transactions, and expectations is determined by collective flows of variables, transactions, and expectations induced by the motion of agents in domain (1). Such financial risk flows result in the motion of agents in domain (1) due to a change of their risk rating.

\subsection{Variations of Agents' Risk Ratings Produce Collective Flows of Variables, Transactions, and Expectations}

Most economic and financial risks like credit, investment, market, or tax policy risks are generated by the activity of economic agents. Any financial activity and development reproduces financial risks. Financial activity without risks is impossible. Changes of agents' risk ratings due to their financial or economic activity and other reasons cause changes of agents' risk coordinates in domain (1). Let us model the change of agents' risks during time $\Delta$ by a certain risk speed vin domain (1). The meaning of risk speed $v$ is simple. For a long time, credit transition matrixes (Bangia et al. 2000; Metz and Cantor 2007; Fitch Ratings 2017; S\&P 2019) have been the subject of intensive studies as tools for default assessment and business cycle modeling. Credit transition matrixes describe the probabilities of transition from the current risk grade to safer or riskier grades over one, two, or more years. Current risk grades have notations such as $A A A, A A, B B$, and $C$ (Moody's Investors Service 2010, 2018). Meanwhile, the "distance" between risk grades $A A A$ and $B B$ makes no sense. The introduction of a continuous risk domain (1) instead of common risk grades allows the define distance between risk grades to be defined. Transitions between two risk grades with distance $x$ during time term $T$ with probabilities $P$ allow the risk velocity $v$ in domain (1) to be defined as (2a): the mean transition risk distance per time $T$. The motion of agents with risk speed $v$ indicates that agents carry their economic and financial variables, transactions, and expectations. For example, if a certain agent provides credits $C$ and moves with speed $v$, then it carries credit flow $\boldsymbol{P}_{C}=C \boldsymbol{v}$. Flows of variables, transactions, and expectations carried by agents due to changes of their risk ratings have important impacts on the economic and financial evolution. Flows of variables and transactions induce continuous changes of financial variables. In particular, this causes continuous changes of the market supply and demand and makes the GE concept of supply-demand equilibrium very questionable. Recent papers on "Rebuilding macroeconomic theory" (Vines and Wills 2018a) do not investigate this issue and we plan to discuss contradictions with the GE concept in a forthcoming work. The collective flows of separate agents define financial flows of variables, transactions, and expectations. Let us explain the meaning of aggregative variables, collective flows, and risk velocities in a more rigorous way.

We describe the macro finance as a system of numerous agents in $n$-dimensional domain (1) and propose that agents at a moment $t$ have risk coordinates $x=\left(x_{1}, \ldots x_{n}\right)$ and velocities $\boldsymbol{v}=\left(v_{1}, \ldots v_{n}\right)$. The velocities $\boldsymbol{v}=\left(v_{1}, \ldots v_{n}\right)$ describe the change of agents' risk coordinates during a time term $\Delta$. Let us explain the relations between the risk velocities and risk transition matrixes (Metz and Cantor 2007; Fitch Ratings 2017; S\&P 2019). We define probability $P_{i j}$ of transition from a risk grade $i$ to a risk grade $j$ during a time term T. Current risk methodologies (Moody's Investors Service 2010; S\&P 2016; Fitch Ratings 2018) note risk grades such as $A A A, A A, B B$, and $C C$, and therefore, difference between two risk grades has no sense. We propose, that risk grades may take values in the domain (1), and the difference between two grades equals the distance $y$ - $x$ between points $x$ and $\mathbf{y}$ of domain (1). We assume, 
that at time $t$ for agent $i$ the function $P_{i}(t ; x, y)$ defines the probability of transition from risk $x$ to $y$ during a time term $\mathrm{T}$. Therefore, we denote the risk velocity $\boldsymbol{v}_{i}$ of agent $i$ as:

$$
\boldsymbol{v}_{i}(t, x)=\frac{1}{T} \int d y P_{i}(t ; x, y)(y-x)=\frac{1}{T}\left[\int d y P_{i}(t ; x, y) y-x\right]
$$

and, for the transition probability $P_{i}(t ; x, y)$ :

$$
\int d y P_{i}(t ; x, y)=1
$$

The transition probability $P_{i}(t ; x, y)$ in domain (1) define mean velocity $\boldsymbol{v}_{i}(2 \mathrm{a})$ of agent $i$. Let us assume that the scale $d<<1$ defines a unit volume $d V$ at point $x$ :

$$
d \ll 1 ; d V=d^{n}
$$

and a volume $d V(2 \mathrm{~b})$ contains many agents. Let us describe only the additive variables of agents and assume that econometric statistics selects "independent" agents. We call agents "independent" if the sum of their additive variables equals the same variable of the entire group. For example, the sum of credits of $k$ agents equals the credits of the group of these $k$ agents. Let us define the additive collective variable $A(t, x)$ at point $x$ as the sum of the variables $A_{i}(t, x)$ of agents $i$ with coordinates in a unit volume $d V(x)(2 \mathrm{~b})$ and then average the sum during term $\Delta$ as:

$$
\begin{gathered}
A(t, x)=\sum_{i \in d V(x) ; \Delta} A_{i}(t, x) \\
\sum_{i \in d V(x) ; \Delta} A_{i}(t, x)=\frac{1}{\Delta} \int_{t}^{t+\Delta} d \tau \sum_{i \in d V(x)} A_{i}(\tau, x)
\end{gathered}
$$

We use $i \in d V(x)$ to denote that the risk coordinates $x$ of agent $i$ belong to a volume $d V(x)$. For brevity, we use left hand sum (4) to denote averaging during time $\Delta$ in a unit volume $d V(x)$. We propose that the scale $\Delta$ is small to compare with the time scales of the problem under consideration, but agents perform a lot of financial transactions between them during time $\Delta$. Time averaging smooth out the changes of agents' variables under numerous transactions during time $\Delta$. We aggregate the values of variables of numerous agents with risk coordinates inside a volume $d V(x)$, smooth their changes during time $\Delta$ and denote the result as a density function of variable at point $x$. The density function $A(t, x)$ describes the collective financial or economic variable at point $x$ in (1). For example, denote $A_{i}(t, x)$ as the credits of an agent $i$. Therefore the density of credits $A(t, x)$ describes the sum of credits issued by all agents with coordinates $x$ inside a unit volume $d V(x)$ and averaged during a time $\Delta$. Then the total credits $A(t)$ in the economy equal the integral (5) over (1):

$$
A(t)=\int d x A(t, x)
$$

Therefore the function $A(t, x)$ (3) can be treated as the financial density of variable $A(t)(5)$ in (1). Now let us introduce the collective flows $\boldsymbol{P}$ and collective velocities $\boldsymbol{v}$. We describe changes of coordinates $x_{i}=\left(x_{1}, \ldots x_{n}\right)$ of an agent $i$ with additive variable $A_{i}(t, x)$ during a time $\Delta$ by velocities $\boldsymbol{v}_{i}$ $=\left(v_{1 i}, \ldots v_{n i}\right)(2 \mathrm{a})$. Thus each agent $i$ carries flow $\boldsymbol{p}_{i A}(t, \boldsymbol{x})$ :

$$
p_{i A}(t, x)=A_{i}(t, x) \boldsymbol{v}_{i}(t, x)
$$

Different agents induce different flows of variable $A$ in different directions with different velocities. Let us collect flows of the variable $A_{i}(t, x)$ in the direction of velocity $\boldsymbol{v}_{i}$ of agent $i$ with coordinates in 
a unit volume $d V(x)(2 b)$ and then average this flow during a time $\Delta$ similar to relations (3) and (4). Let us define the collective flow $\boldsymbol{P}_{A}(t, x)$ of variable $A(t, x)$ as:

$$
\boldsymbol{P}_{A}(t, x)=\sum_{i \in d V(x) ; \Delta} A_{i}(t, x) \boldsymbol{v}_{i}(t, x)
$$

Similar to (5) the integral of (7) by $d x$ over (1) defines the macro flow $\boldsymbol{P}_{A}(t)$ of the variable $A(t)$ :

$$
\boldsymbol{P}_{A}(t)=\int d x \boldsymbol{P}_{A}(t, x)
$$

The flow $\boldsymbol{P}_{A}(t, x)$ (7) of the variable $A(t, x)$ (3) defines the collective velocity $\boldsymbol{v}_{A}(t, x)$ of the variable $A(t, x)$ as:

$$
\boldsymbol{P}_{A}(t, x)=A(t, x) \boldsymbol{v}_{A}(t, x)
$$

Therefore (9) describes the flow $\boldsymbol{P}_{A}(t, x)$ of the variable $A(t, x)$ with velocity $\boldsymbol{v}_{A}(t, x)$. Relations (5) and (8) define the macro velocity $\boldsymbol{v}_{A}(t)$ of macro variable $A(t)$ in (1) as:

$$
\boldsymbol{P}_{A}(t)=A(t) \boldsymbol{v}_{A}(t)
$$

One can obtain relations (8) and (10) as a sum of flows (6) of all agents of the entire economics. Let us mention that due to (3), (5), (7)-(10) the velocity $\boldsymbol{v}_{A}(t)$ is not equals to the integral of velocity $\boldsymbol{v}_{A}(t, x)$ over domain (1). Due to (3)-(10) different variables $A$ define different collective flows $\boldsymbol{P}_{A}(t, x)$ with different velocities $\boldsymbol{v}_{A}(t, x)$. In other words-different additive variables $A(t, x)$ in (1) have different velocities $\boldsymbol{v}_{A}(t, x)$. For example, the flow $\boldsymbol{P}_{C}(t, x)$ of credits $C(t, x)$ has the velocity $\boldsymbol{v}_{C}(t, x)$ different from the velocity $\boldsymbol{v}_{L}(t, x)$ of the collective flow $\boldsymbol{P}_{L}(t, x)$ of loans $L(t, x)$ or from collective velocity $\boldsymbol{v}_{I}(t, x)$ that describes flow $\boldsymbol{P}_{I}(t, x)$ of the investment $I(t, x)$ in (1). The flows (8) and (10) for different variables are also different. For example, flows $P_{S}(t)$ and velocities $v_{S}(t, x)$ of the market supply are different from flows $P_{D}(t)$ and velocities $\boldsymbol{v}_{D}(t, x)$ of the market demand for any commodities, assets, or goods and any markets. This causes permanent changes of supply and demand and makes the existence of imaginable market supply-demand equilibrium states very doubtful. There have been no assessments of the time scales that establish market supply-demand equilibrium states. Risk actions and the impact of financial risk flows disturb any equilibrium states and make GE as a concept too questionable. We propose that further research on the applicability of the GE concept is required. Macro models should describe dynamics and mutual interactions between numerous financial and economic variables, market transactions, expectations, and their flows. It should be highlighted that the properties of economic and financial flows are completely different from the properties of any physical flows.

As we show below, similar considerations help to define collective flows of transactions and expectations. To outline the impact of collective flows of variables, transactions, and expectations on macro finance, equations which govern collective variables, transactions, and expectations can be considered as functions of risk coordinates. All equations have similar form and we derive them for the credit density function $C(t, x)$ as example.

The credit density function $C(t, x)$ (3) and (4) describes collective credits issued by agents with coordinates inside a small volume $d V$ at a point $x$. The motion of agents inside a volume $d V$ induces collective credit flows $\boldsymbol{P}_{C}(t, x)=C(t, x) \boldsymbol{v}(t, x)(7)$ and (9). The function $\boldsymbol{v}(t, x)$ describes velocity of the credit density flow $C(t, x) \boldsymbol{v}(t, x)$. To describe changes of the credit density function $C(t, x)$ during time $d t$ in a small volume $d V$ on (1) let us take into account two factors. The first one describes changes of $C(t, x)$ in a time $d t$ in a small volume $d V$ :

$$
\int d V \frac{\partial}{\partial t} C(t, x)
$$

The second factor describes changes induced by the credit flow $\boldsymbol{P}_{C}=C \boldsymbol{v}$ of agents that may flow in- or flow out- of a small volume $d V$ during a time $d t$. Agents that flow in- a volume $d V$ during $d t$ with 
the credit flow $\boldsymbol{P}_{C}=C \boldsymbol{v}$ increase the credit density function $C(t, x)$ in a volume $d V$ and agents that flow out of the volume $d V$ with credit flow $\boldsymbol{P}_{C}=C v$ decrease the credit density function $C(t, x)$. The balance of credit in- and out-flows equals the integral of the credit flow $\boldsymbol{P}_{C}(t, x)=C(t, x) \boldsymbol{v}(t, x)$ over the surface of a volume $d V$ :

$$
\oint d s \boldsymbol{P}_{C}(t, x)=\oint d s C(t, x) v(t, x)
$$

According to well-known divergence Gauss' Theorem (Strauss 2008, p. 179), the surface integral of the flows equals the volume integral of the flows' divergence over a small volume $d V$ :

$$
\oint d s C(t, x) v(t, x)=\int d V \nabla \cdot(C(t, x) v(t, x))
$$

Hence the total change of the credit density function during a time $d t$ in a small volume $d V$ equals:

$$
\int d V\left[\frac{\partial}{\partial t} C(t, x)+\nabla \cdot(C(t, x) v(t, x))\right]
$$

A volume $d V$ is an arbitrary small and thus equations on $C(t, x)$ (Olkhov 2016a, 2016b, 2017a) take form:

$$
\frac{\partial}{\partial t} C(t, x)+\nabla \cdot(C(t, x) v(t, x))=F_{C}(t, x)
$$

The function $F_{C}(t, x)$ in the right side (11b) describes the impact of factors defined by variables, transactions, or expectations and their flows on evolution of the credit density function $C(t, x)$. The Equation (11b) depends on credit flow $\boldsymbol{P}_{C}(t, x)=C(t, x) \boldsymbol{v}(t, x)$ and hence one should derive equation on it. The same considerations permit derive equations on the flow $\boldsymbol{P}_{C}(t, x)=C(t, x) \boldsymbol{v}(t, x)$ as:

$$
\frac{\partial}{\partial t} \boldsymbol{P}_{C}(t, x)+\nabla \cdot\left(\boldsymbol{P}_{C}(t, x) \boldsymbol{v}(t, x)\right)=G_{C}(t, x)
$$

The function $G_{C}(t, x)$ in (11c) describes factors defined by variables, transactions, and expectations and their flows those impact evolution of the credit flow $\boldsymbol{P}_{C}(t, \boldsymbol{x})$. Due to (5) the integral by $d x$ of (11b) over (1):

$$
\frac{d}{d t} C(t)+\int d x \nabla \cdot(C(t, x) v(t, x))=\int d x F_{C}(t, x)=F_{C}(t)
$$

Due to (11a) the integral in the left side (11d) equals zero as no agents, in- or out- flows exist on surface outside of domain (1). Therefore (11d) takes form of ordinary differential equation:

$$
\frac{d}{d t} C(t)=F_{C}(t)
$$

The complexities of (11e) are hidden by the function $F_{C}(t)$ determined by the integral in (11d). The function $F_{C}(t, x)$ may depend on many variables, transactions, expectations, and their flows and may be a very complex function. Thus time evolution of the collective variables like the macro credits $C(t)$ may depend on the hidden dynamics of variables, transactions, and expectations and their flows in domain (1). Due to (8), (10) and (11a) the integral by $d x$ for Equations (11c) over domain (1) defines ordinary differential equation on credit flow $\boldsymbol{P}_{C}(t)$ :

$$
\frac{d}{d t} \boldsymbol{P}_{C}(t)=\int d x G_{C}(t, x)=G_{C}(t)
$$

The function $G_{C}(t)$ in (11f) may be as complex as function $F_{C}(t)(11 \mathrm{~d})$. Equations similar to (11b), (11c), (11e) and (11f) are valid for other additive variables such as investment, loans, demand, and supply, and their flows. Each aggregate variable $A(t)$ as a function of time has a different velocity $\boldsymbol{v}(t)$. Macroevolution requires a description of the motion of numerous financial variables with different 
velocities in (1) and that is a tough problem. The meaning of (11f) will now be presented. Velocity $\boldsymbol{v}(t)$ of credit flow $\boldsymbol{P}_{C}(t)=C(t) \boldsymbol{v}(t)$ describes the motion of credits $C(t)$ in (1). Economic domain (1) is bounded along each risk axis by the most secure and most risky grades [0,1]. Therefore, the motion of credits $C(t)$ with velocity $\boldsymbol{v}(t)$ along each risk axis from a secure to risky direction should change by the opposite motion from a risky to secure area. Hence, credit velocity $\boldsymbol{v}(t)$ should fluctuate in time and such fluctuations describe credit cycles. Similar fluctuations describe cycles of GDP, investment, etc.

There are some consequences of our model. As we mentioned above, equations similar to (11b) and (11c) describe density functions and flows of numerous financial variables, transactions, and expectations. Therefore, equations similar to (11b) and (11c) define macro models for each selected set of variables, transactions, and expectations. The model can be determined by a set of $k$ different transactions like credit, investment, and buy-sell transactions. Each transaction defines a change of variables of sellers and buyers. For example, a credit transaction changes the value of credits provided by the creditor (seller) and amount of loans received by borrowers (buyers). Therefore, $k$ transactions change $2 k$ additive variables. Each transaction can be performed under different expectations. It can be assumed that $k$ transactions are performed under $W$ expectations. To develop a self-consistent model that describes the macro model determined by $2 k$ additive variables and $k$ transactions, one should assume that all $W$ expectations are determined by $2 k$ additive variables, $k$ selected transactions, and their flows. In particular, $W$ expectations should depend on $2 k$ additive variables or non-additive variables that can be determined by $2 k$ additive variables and their flows. If some expectations depend on exogenous factors, then evolution of the macroeconomic model reflects the action of exogenous properties. Exogenous expectations transfer the impact of exogenous factors on macro financial dynamics.

The importance of expectations is not reduced by their role as a transmitter of exogenous shocks. As we argued above, expectations may depend on flows of variables, transactions, and other expectations. Expectations formed by financial flows become key factors that determine the impact of flows on financial evolution. Dynamics of flows of variables, transactions, and expectations and their mutual interactions in (1) establish a very complex picture. For example, flows in domain (1) generate business, credit, and investment cycles that describe slow oscillations of macro financial variables. On the other hand, perturbations of flows produce generation, propagation, and interaction of waves of disturbances of financial variables, transactions, and expectations, and induce fast oscillations of financial parameters. We apply our methods to study the approximations based on equations similar to (11b) and (11c) that describe a "simplified" model interactions between two variables (Olkhov 2016a, 2016b), between two transactions (Olkhov 2018a), model the business cycles (Olkhov 2017c, 2019), and wave propagation of disturbances of financial variables (Olkhov 2016a, 2016b, 2017a), and transactions (Olkhov 2018a), and surface-like waves (Olkhov 2017b) in domain (1). In the Section 5 we apply equations similar to (11b), (11c) to model price fluctuations induced by interactions between transactions and numerous expectations.

\section{Market Transactions as Functions of Risk}

Let us assume, that agent $i$ at point $x$ sell the amount $Q_{i j}$ of variable $E$ to agent $j$ at point $\mathbf{y}$. Variable $E$ may make sense of commodities, credits, investment, assets, service, etc. For example, agent $i$ provides the credits $C$ to agent $j$. Such a transaction between agents $i$ and $j$ changes the amount of credits $C$ provided by $i$ and the amount of loans $L$ received by $j$. The transaction of amount $Q_{i j} \operatorname{cost}$ certain value $C_{i j}$ that should be paid by the agent $j$ as buyer to the agent $i$ as seller. Therefore each transaction defines two variables-the amount $Q_{i j}$ and the cost $C_{i j}$ and these variables define price $p_{i j}$ of the financial transaction of variable $E$. For the agent $i$ with risk coordinates $x$ and the agent $j$ with coordinates $y$ at moment $t$ we define the transaction $b s_{i j}(t, x, y)$ with the amount $Q_{i j}(t, x, y)$ and cost $C_{i j}(t, x, y)$ as:

$$
\boldsymbol{b s}_{i j}(t, \boldsymbol{z})=\left(Q_{i j}(t, \boldsymbol{z}) ; C_{i j}(t, \boldsymbol{z})\right) ; \boldsymbol{z}=(\boldsymbol{x}, \boldsymbol{y})
$$


Then the price $p_{i j}(t, z)$ of the transaction (12a) takes obvious form:

$$
p_{i j}(t, z)=C_{i j}(t, z) / Q_{i j}(t, z) ; z=(x, y)
$$

We use bold font for the transaction $\boldsymbol{b s}_{i j}(t, z), z=(x, y)$ to underline that each transaction defines two additive variables-the amount $Q_{i j}$ and the cost $C_{i j}$ of economic or financial variable $E$. It is obvious, that $\operatorname{cost} C_{i j}$ may be transferred later then amount $Q_{i j}$ or vice versa but for simplicity as first approximation we assume that it is done simultaneously. Each transaction takes a certain time $d t$ and we consider transactions as a rate or speed of change of corresponding variable $E$ for agents involved into the transaction. For example, all transactions of agent $i$ at a moment $t$ during time $[0, t]$ define change of variable $E$ (Steel, Energy, Shares, Credits, Assets etc.) owned by agent $i$ during a period $[0, t]$. Now, let us move from description of transactions $b s_{i j}(t, x, y)$ between separate agents $i$ at $x$ and $j$ at $y$ to description of collective transactions $\boldsymbol{B S}(t, x, y)$ between points $x$ and $\boldsymbol{y}$.

Let us assume that agents in (1) at a moment $t$ have coordinates $x=\left(x_{1}, \ldots x_{n}\right)$ and risk velocities $v$ $=\left(v_{1}, \ldots v_{n}\right)$. The transactions between agents with risk coordinates $x$ and agents with risk coordinates $y$ are determined on $2 n$-dimensional economic domain, $z=(x, y)$ :

$$
\begin{gathered}
z=(x, y) ; \boldsymbol{x}=\left(x_{1} \ldots x_{n}\right) ; \boldsymbol{y}=\left(y_{1} \ldots y_{n}\right) \\
0 \leq x_{i} \leq 1 ; 0 \leq y_{i} \leq 1, i=1, \ldots n
\end{gathered}
$$

Relations (12c) and (12d) define $2 n$-dimensional economic domain and pairs of agents with coordinates $z=(x, y)$ fill this domain. Let us take a unit volume $d V(z)$

$$
d V(\boldsymbol{z})=d V(\boldsymbol{x}) d V(\boldsymbol{y}) ; d V(\boldsymbol{x})=d^{n} ; d V(\boldsymbol{x})=d^{n} ; \boldsymbol{z}=(\boldsymbol{x}, \boldsymbol{y})
$$

and assume that $d V(x)$ and $d V(y)$ obey relations (2a) and their scales $d<<1$. Let us assume that each unit volume $d V(x)$ and $d V(y)$ contain a lot of agents with risk coordinates inside $d V(x)$ and $d V(y)$ and during time $\Delta$ agents inside $d V(x)$ and $d V(y)$ perform a lot of mutual transactions. Let us define the collective transaction $\boldsymbol{B S}(t, x, y)$ between points $\boldsymbol{x}$ and $\boldsymbol{y}$ as a sum of all transactions of agents $i$ with coordinates in a unit volume $d V(\boldsymbol{x})$ and agents $j$ with coordinates in a unit volume $d V(\boldsymbol{y})(12 \mathrm{e})$ and then average this sum during the term $\Delta$ similar to (3) and (4) as:

$$
\begin{gathered}
\boldsymbol{B S}(t, \boldsymbol{x}, \boldsymbol{y})=\sum_{i \in d V(\boldsymbol{x}) ; j \in d V(\boldsymbol{y}) ; \Delta} \boldsymbol{b} \boldsymbol{s}_{i, j}(t, \boldsymbol{x}, \boldsymbol{y}) \\
\sum_{i \in d V(\boldsymbol{x}) ; \Delta} \boldsymbol{b} \boldsymbol{s}_{i, j}(t, \boldsymbol{x}, \boldsymbol{y})=\frac{1}{\Delta} \int_{t}^{t+\Delta} d \tau \sum_{i \in d V(\boldsymbol{x}) ; j \in d V(\boldsymbol{y})} \boldsymbol{b} \boldsymbol{s}_{i, j}(\tau, \boldsymbol{x}, \boldsymbol{y}) \\
\boldsymbol{B S}(t, \boldsymbol{z})=(Q(t, \boldsymbol{z}) ; C(t, \boldsymbol{z})) ; \boldsymbol{z}=(\boldsymbol{x}, \boldsymbol{y}) \\
Q(t, \boldsymbol{z})=\sum_{i \in d V(\boldsymbol{x}) ; j \in d V(\boldsymbol{y}) ; \Delta} \sum_{k_{1}} Q_{i j}(t, \boldsymbol{z}) \\
C(t, \boldsymbol{z})=\sum_{i \in d V(\boldsymbol{x}) ; j \in d V(\boldsymbol{y}) ; \Delta} \sum_{k_{2}} C_{i j}(t, \boldsymbol{z})
\end{gathered}
$$

We take the price $p(t, z)$ of the transaction $\boldsymbol{B S}(t, z)$ as:

$$
C(t, z)=p(t, z) Q(t, z) ; z=(x, y)
$$

The integral of transaction $B S(t, z)(12 \mathrm{~h})$ by $d y$ over the economic domain (12c) and (12d) defines all sells $B S(t, x)$ of variable $E$ performed by agents inside a unit volume $d V(x)$ at $x$

$$
\boldsymbol{B S}(t, x)=\left(Q_{S}(t, x) ; C_{S}(t, x)\right)
$$




$$
Q_{S}(t, x)=\int d y Q(t, x, y) ; C_{S}(t, x)=\int d y C(t, x, y)
$$

Relations (13a) and (13b) define the price $p_{S}(t, x)$ of sellers for the transactions with variable $E$ from $x$ :

$$
C_{S}(t, x)=p_{S}(t, x) Q_{S}(t, x)
$$

The integral of transaction $\boldsymbol{B S}(t, z)(12 \mathrm{~h})$ by $d \boldsymbol{x}$ over (12c) and (12d) defines the buyers' price $p_{B}(t, \boldsymbol{y})$ at $y$ :

$$
C_{B}(t, y)=\int d x C(t, x, y)=p_{B}(t, y) Q_{B}(t, y) ; Q_{B}(t, y)=\int d x Q(t, x, y)
$$

The integral of the transaction $\boldsymbol{B S}(t, z)(12 \mathrm{~h})$ by $d x d y$ over (12c) and (12d) define the trading volume $Q(t)$, the $\operatorname{cost} C(t)$, and the price $p(t)$ of transactions $B S(t)$ in economy at a moment $t$ :

$$
\begin{gathered}
B S(t)=(Q(t) ; C(t)) \\
C(t)=\int d x d y C(t, x, y)=p(t) Q(t) ; Q(t)=\int d x d y Q(t, x, y)
\end{gathered}
$$

For example, if $C I(t)$ equals the amount of cumulative investment made in economy during a term $[0, t]$ and $Q(t, x, y)$ - the amount of investment transactions $B S(t, x, y)(12 \mathrm{f})$ made from $x$ to $y$ during a time term $d t$ then from (12f):

$$
\frac{d}{d t} C I(t)=Q(t)=\int d x d y Q(t, x, y)
$$

Hence, the transactions define time derivative of cumulative macro variables like investment, credits, etc. Let us note $B S(t, z)$ as a transaction density function on $2 n$-dimensional domain $(12 \mathrm{c})$ and (12d) similar to a variable density function $A(t, x)$ (3) and (4) on (1). Relations (12f)-(13d) demonstrate that different aggregations describe different price of transactions. Thus, different aggregations of the price have a different impact on the price evolutions and fluctuations.

Similar to (6) and (7), let us introduce the transactions flows that are induced by changes of risk coordinates of agents at point $x$ and $y$. Indeed, a motion of agents due to changes of their risk coordinates induces flows of the transactions that change the amount and cost of the transactions in a small volume (12c)-(12e). Let us define the flows $p_{i j}(t, z)(14 \mathrm{a})$ and (14b) of the transactions $b s_{i j}(t, z)$ between agents $i$ and $j$ similar to (6):

$$
\begin{aligned}
& \boldsymbol{p}_{i j}(t, \boldsymbol{z})=\left(\boldsymbol{p}_{\mathrm{Qij}}(t, \boldsymbol{z}), \boldsymbol{p}_{\mathrm{C} i j}(t, \boldsymbol{z})\right) \\
& p_{\text {Qij }}(t, z)=\left(p_{\text {Qijx }}(t, z) ; p_{\text {Qijy }}(t, z)\right) ; p_{C i j}(t, z)=\left(p_{C i j x}(t, z) ; p_{C i j y}(t, z)\right) \\
& \boldsymbol{p}_{\mathrm{Qijx}}(t, \boldsymbol{z})=Q_{i, j}(t, \boldsymbol{z}) \boldsymbol{v}_{i x}(t, \boldsymbol{x}) ; \boldsymbol{p}_{\mathrm{Qijy}}(t, \boldsymbol{z})=Q_{i, j}(t, \boldsymbol{z}) \boldsymbol{v}_{j y}(t, \boldsymbol{y}) \\
& \boldsymbol{p}_{C i j x}(t, \boldsymbol{z})=C_{i, j}(t, \boldsymbol{z}) \boldsymbol{v}_{i x}(t, \boldsymbol{x}) ; \boldsymbol{p}_{C i j y}(t, \boldsymbol{z})=C_{i, j}(t, \boldsymbol{z}) \boldsymbol{v}_{j y}(t, \boldsymbol{y})
\end{aligned}
$$

The flows $p_{i j}(t, z)(14 \mathrm{a})$ define the flows $p_{Q i j}(t, z)(14 \mathrm{c})$ that carry the amount $Q_{i j}$ and the flows $p_{Q i j}(t, z)(14 \mathrm{~d})$ that carry the $\operatorname{cost} C_{i j}$ of the transaction $\boldsymbol{b} s_{i j}(t, z)$ (12a). The cumulative flows $\boldsymbol{P}(t, z)$ over all agents $i$ at $x$ inside $d V(x)$ and all agents $j$ at $y$ inside $d V(y)$ define the transactions flows between points $x$ and $y$ similar to (7) as:

$$
\begin{gathered}
\boldsymbol{P}(t, \boldsymbol{z})=\left(\boldsymbol{P}_{Q}(t, \boldsymbol{z}), \boldsymbol{P}_{C}(t, \boldsymbol{z})\right) ; \boldsymbol{z}=(\boldsymbol{x}, \boldsymbol{y}) \\
\boldsymbol{P}_{Q}(t, \boldsymbol{z})=\sum_{i \in d V(\boldsymbol{x}) ; j \in d V(\boldsymbol{y}) \Delta} \boldsymbol{p}_{Q i j}(t, \boldsymbol{z}) ; \boldsymbol{P}_{C}(t, \boldsymbol{z})=\sum_{i \in d V(\boldsymbol{x}) ; j \in d V(\boldsymbol{y}) \Delta} \boldsymbol{p}_{C i j}(t, \boldsymbol{z}) \\
\boldsymbol{P}_{Q}(t, \boldsymbol{z})=\left(\boldsymbol{P}_{x Q}(t, \boldsymbol{z}) ; \boldsymbol{P}_{y Q}(t, \boldsymbol{z})\right) ; \boldsymbol{P}_{C}(t, \boldsymbol{z})=\left(\boldsymbol{P}_{x C}(t, \boldsymbol{z}) ; \boldsymbol{P}_{y C}(t, \boldsymbol{z})\right)
\end{gathered}
$$




$$
\begin{gathered}
\boldsymbol{P}_{x Q}(t, \boldsymbol{z})=\sum_{i \in d V(x) ; j \in d V(y) \Delta} Q_{i j}(t, \boldsymbol{z}) \boldsymbol{v}_{i}(t, \boldsymbol{x})=Q(t, \boldsymbol{z}) \boldsymbol{v}_{x Q}(t, \boldsymbol{z}) \\
\boldsymbol{P}_{y Q}(t, \boldsymbol{z})=\sum_{i \in d V(x) ; j \in d V(y) \Delta} Q_{i j}(t, \boldsymbol{z}) \boldsymbol{v}_{j}(t, \boldsymbol{y})=Q(t, \boldsymbol{z}) \boldsymbol{v}_{y Q}(t, \boldsymbol{z}) \\
\boldsymbol{P}_{x C}(t, \boldsymbol{z})=\sum_{i \in d V(\boldsymbol{x}) ; j \in d V(y) \Delta} C_{i j}(t, \boldsymbol{z}) \boldsymbol{v}_{i}(t, \boldsymbol{x})=C(t, \boldsymbol{z}) \boldsymbol{v}_{x C}(t, \boldsymbol{z}) \\
\boldsymbol{P}_{y C}(t, \boldsymbol{z})=\sum_{i \in d V(x) ; j \in d V(\boldsymbol{y}) \Delta} C_{i j}(t, \boldsymbol{z}) \boldsymbol{v}_{j}(t, \boldsymbol{y})=C(t, \boldsymbol{z}) \boldsymbol{v}_{y C}(t, \boldsymbol{z}) \\
\boldsymbol{v}(t, \boldsymbol{z})=\left(\boldsymbol{v}_{Q}(t, \boldsymbol{z}) ; \boldsymbol{v}_{C}(t, \boldsymbol{z})\right) \\
\boldsymbol{v}_{Q}(t, \boldsymbol{z})=\left(\boldsymbol{v}_{x Q}(t, \boldsymbol{z}) ; \boldsymbol{v}_{y Q}(t, \boldsymbol{z})\right) ; \boldsymbol{v}_{C}(t, \boldsymbol{z})=\left(\boldsymbol{v}_{x C}(t, \boldsymbol{z}) ; \boldsymbol{v}_{y C}(t, \boldsymbol{z})\right)
\end{gathered}
$$

The flows of transactions $\boldsymbol{P}(t, z)(15 \mathrm{a})-(15 \mathrm{f})$ between points $\boldsymbol{x}$ and $\boldsymbol{y}$ describe the amounts of transactions $\boldsymbol{B S}(t, z)(13 \mathrm{a})$ carried by transactions velocities $\boldsymbol{v}(t, z)(15 \mathrm{~g})-(15 \mathrm{i})$ through $2 n$-dimensional domain (12c) and (12d). Let us underline that velocities $v_{Q}(t, z)(15 \mathrm{~h})$ that define the motion of amount of transactions may be different from velocities $v_{C}(t, z)(15 i)$ that describe the motion of transactions costs. These distinctions add additional perturbations for price of the transactions (12k). Similar to (8) and (9) integrals of the flows $\boldsymbol{P}(t, z)(15 \mathrm{a})-(15 \mathrm{f})$ and (15g)-(15i) over (12c) and (12d) by $d x d y$ we define the macro flows of transactions $\boldsymbol{B S}(t)(4.1)$ with velocity $\boldsymbol{v}(t)$ as:

$$
\begin{gathered}
\boldsymbol{P}(t)=\left(\boldsymbol{P}_{Q}(t) ; \boldsymbol{P}_{C}(t)\right) \\
\boldsymbol{P}_{Q}(t)=Q(t) \boldsymbol{v}_{Q}(t)=\int d z Q(t, z) \boldsymbol{v}_{Q}(t, \boldsymbol{y}) \\
\boldsymbol{P}_{C}(t)=C(t) \boldsymbol{v}_{C}(t)=\int d \boldsymbol{z} C(t, \boldsymbol{z}) \boldsymbol{v}_{C}(t, \boldsymbol{y}) \\
\boldsymbol{v}(t)=\left(\boldsymbol{v}_{Q}(t) ; \boldsymbol{v}_{C}(t)\right) \\
\boldsymbol{v}_{Q}(t, \boldsymbol{z})=\left(\boldsymbol{v}_{x Q}(t) ; \boldsymbol{v}_{y Q}(t)\right) ; \boldsymbol{v}_{C}(t, \boldsymbol{z})=\left(\boldsymbol{v}_{x C}(t) ; \boldsymbol{v}_{y C}(t)\right)
\end{gathered}
$$

For example, let us take $\boldsymbol{B S}(t)$ as investment transactions with an amount of investment $Q(t)$ in the economy at moment $t$. Then, the relations (16b) describe the flow of the amount of investment with velocity $v_{Q}(t)$ on (12c) and (12d). Components $\boldsymbol{v}_{x Q}(t)$ and $\boldsymbol{v}_{y Q}(t)$ describe the motion of collective investors and recipients of investments. Positive or negative values of components of velocity $\boldsymbol{v}_{x i Q}(t)$ along axis $x_{i}$ of (12c) and (12d) describe the motion of investors in risky or safer directions. Positive values of components of velocity $\boldsymbol{v}_{y j Q}(t)$ along axis $y_{j}$ of (12c) and (12d) describe the motion of recipients of investments in a risky direction and negative $\boldsymbol{v}_{y j}(t)$ describes a decline of risks of recipients of investments along axis $y_{j}$. Collective investors and recipients of investments may only move inside the bounded domain (12c) and (12d). Therefore, velocities (16d) and (16e) cannot be constant and must change their signature and fluctuate as borders of domain (12c) and (12d) reduce the motion along each risk axis. Fluctuations of velocities (16d) and (16e) describe the motion of investors and recipients of investments from safer to risky areas and back from risky to safer areas, and thus describe investment cycles. Credit transactions, buy-sell transactions, etc., induce similar macro transactions flows (16a)-(16e) and describe corresponding credit cycles, buy-sell cycles, etc., (Olkhov 2017c, 2019).

The relations (12f)-(12j) and (14a)-(15i) allow derive equations on the transactions $B S(t, z)$ and transactions flows $\boldsymbol{P}(t, z), z=(x, y)$ on $2 n$-dimensional domain (12c) and (12d) similar to the Equations $(11 b)$ and (11c) on density and flows of variables (3), (4) and (7). To derive equations on transactions density $\boldsymbol{B S}(t, z)(12 \mathrm{~h})-(12 \mathrm{j})$ and flows $\boldsymbol{P}(t, z)(15 \mathrm{a})-(15 \mathrm{i})$, let us describe their changes in a small unit volume $d V(z)$ (12c)-(12e). Let us take equations on the amount of transactions $Q$ (12i) and its flows $\boldsymbol{P}_{Q}$. 
Equations on cost of transaction take similar form. Two factors change the amount of transaction $Q(t, z)$ in a unit volume $d V(z)$. The first one change $Q(t, z)$ in time as:

$$
\int d z \frac{\partial}{\partial t} Q(t, z)
$$

The second factor describes change of $Q(t, z)$ due to flows $\boldsymbol{P}_{Q}(t, z)$ : the amount of $Q(t, z)$ in a unit volume $d V(z)$ (12c)-(12e) can grow up or decrease due to in- or out-flows $\boldsymbol{P}_{Q}(t, z)$ during time $d t$. If inflows $\boldsymbol{P}_{Q}(t, z)$ exceed out- flows then $Q(t, z)$ grow up in a volume $d V(z)$. To calculate the balance of inand out-flows let us take the integral of flow $\boldsymbol{P}_{Q}(t, z)$ over the surface of $d V(z)$ :

$$
\oint d s \boldsymbol{P}_{Q}(t, z)=\oint d s Q(t, z) v_{Q}(t, z)
$$

Due to the divergence theorem (Strauss 2008, p. 179) the surface integral (17b) of the flow $\boldsymbol{P}_{Q}(t, z)$ $=Q(t, z) \boldsymbol{v}_{Q}(t, z)$ equals its volume integral by divergence of the flow:

$$
\oint d s Q(t, z) v_{Q}(t, z)=\int d z \nabla \cdot\left(Q(t, z) v_{Q}(t, z)\right)
$$

The relations (17a)-(17c) give total change of the amount of transactions $Q(t, z)$ in $d V(z)$ :

$$
\int d z\left[\frac{\partial}{\partial t} Q(t, z)+\nabla \cdot\left(Q(t, z) v_{Q}(t, z)\right)\right]
$$

As a unit volume $d V(z)$ is an arbitrary, one can take equations on $Q(t, z)$ as

$$
\frac{\partial}{\partial t} Q(t, z)+\nabla \cdot\left(Q(t, z) v_{Q}(t, z)\right)=F(t, z)
$$

The same considerations are valid for the flow $\boldsymbol{P}_{Q}(t, z)$ :

$$
\frac{\partial}{\partial t} \boldsymbol{P}_{Q}(t, z)+\nabla \cdot\left(\boldsymbol{P}_{Q}(t, z) \boldsymbol{v}_{Q}(t, z)\right)=G(t, z)
$$

Similar to (11d) and (11e) the integrals of (17d) and (17e) by $d z=(d x, d y)$ over domain (12c) and (12d) give:

$$
\begin{gathered}
\int d z\left[\frac{\partial}{\partial t} Q(t, z)+\nabla \cdot\left(Q(t, z) v_{Q}(t, z)\right)\right]=\frac{d}{d t} Q(t)=F(t)=\int d z F(t, z) \\
\int d z\left[\frac{\partial}{\partial t} \boldsymbol{P}_{Q}(t, z)+\nabla \cdot\left(\boldsymbol{P}_{Q}(t, \boldsymbol{z}) \boldsymbol{v}_{Q}(t, \boldsymbol{z})\right)\right]=\frac{d}{d t} \boldsymbol{P}_{Q}(t)=\boldsymbol{G}(t)=\int d z \boldsymbol{G}(t, \boldsymbol{z})
\end{gathered}
$$

The relations (18a) and (18b) illustrate that operators in the left hand of (17d) and (17e) for $Q(t, z)$ and the flows $\boldsymbol{P}_{Q}(t, z), z=(x, y)$ on the $2 n$-dimensional domain (12c), (12d) play role alike to ordinary derivative by time $t$ for the amount of transactions $Q(t)$ (12i) and (18a) and flows $\boldsymbol{P}_{Q}(t)(15 \mathrm{c})$ and (18b). Equations similar to (17d), (17e) and (18a), (18b) are valid for the cost of transactions $C(t, z)$ and cost flows $\boldsymbol{P}_{C}(t, z), z=(x, y)$, but velocities $\boldsymbol{v}_{C}(t, z)$ of the cost flows $\boldsymbol{P}_{C}(t, z)$ are different from velocities $\boldsymbol{v}_{Q}(t, z)$ of amount flows $\boldsymbol{P}_{Q}(t, z)$. Each component of each transaction has a different velocity and is described by different operators (17d), (17e) and (18a), (18b) with a different functions $F(t, z)$ and $G(t, z)$. Such a variety of flows and velocities in the domain (12c), (12d) establishes a very complex picture of economic and financial processes and their evolution. The form of functions $F(t, z)$ and $G(t, z)$ and the questions relating to what factors impact equations on transactions (17d), (17e) and (18a), (18b), reflect the main complexity for modeling financial transactions and variables.

Various expectations impact the way in which agents perform transactions $\boldsymbol{B S}(t, z)$ with other agents. Equations (17d) and (17e) define the evolution of the amount $Q$ (12i) of transactions and similar 
equations describe the $\operatorname{cost} C(12 \mathrm{j})$ of transactions. We propose that functions $F(t, z)$ and $G(t, z)$ on the right-hand side of (17d) and (17e) describe the action of expectations of agents involved in transactions $B S(t, z)$. Expectations may depend on economic or financial variables, transactions, expectations of other agents, market and tax regulation, technology trends, and forecasts. This permits the study of the evolution of financial systems in different approximations.

Expectations are very numerous and different. Agents may go into the same transactions under various expectations. For example, different agents at point $x$ may make decisions on amount $Q$ (12i) of the same transaction under inflation expectations, return expectations, professional macroeconomic forecasters (these expectations are mentioned by (Manski 2017)), etc. It is clear that the composition of different expectations as inflation, return, or professional macroeconomic forecasters does not help to establish collective expectations that may explain the aggregate amount $Q(t, z)(12 \mathrm{i})$ of transactions made from point $x$ to point $y, z=(x, y)$. To describe the collective impact of heterogeneous expectations, let us introduce definitions of expectations similar to macro variables and transactions.

\section{Expectations as Functions of Risk}

Expectations are the most "etheric" substance of economics and finance. Expectations are treated as factors that govern financial transactions, prices, and returns at least since Keynes (1936), Muth (1961), and Lucas (1972) and in numerous publications (Sargent and Wallace 1976; Hansen and Sargent 1979; Kydland and Prescott 1980; Blume and Easley 1984; Brock and Hommes 1998; Manski 2004; Brunnermeier and Parker 2005; Dominitz and Manski 2005; Klaauw et al. 2008; Janžek and Ziherl 2013; Greenwood and Shleifer 2014; Lof 2014; Manski 2017; Thaler 2018).

Expectations concern inflation and demand, exchange and bank rates, price trends, etc. There are many studies on expectations measurements (Manski 2004; Dominitz and Manski 2005; Klaauw et al. 2008; Stangl 2009; Janžek and Ziherl 2013; Manski 2017; Tanaka et al. 2018). Due to Manski (2004) "It would be better to measure expectations as—subjective probabilities". Dominitz and Manski (2005) "analyze probabilistic expectations of equity returns". Stangl (2009) suggests that "Visual Analog Scale (VAS) enables scores between categories, and the respondent can express not only the direction of his attitude but also its magnitude on a 1-to-100-point scale, which comes close to an interval scale measurement". The measurement of such "etheric" substances as expectations of separate agents is a really tough problem. Let us propose that it is possible to measure the expectations of separate agents. How can we establish collective expectations that govern transactions made by agents at point $x$ ? Indeed, aggregate transactions (12f)-(12j) are performed under collective expectations of agents in the domain (12c) and (12d) at point $z$ in a unit volume (12e). It is impossible to collect different expectations like "inflation expectations, return, professional macroeconomic forecasters" in one aggregate expectation. To define collective expectations, let us simplify the problem. It can be assumed that all different expectations are measured as an index. It is clear that the scale of the index is not important. The measure of expectations may have values between 0 and 100 or 0 and 1 . All expectations are measured by the same measure with the same scale. For certainty, interval $[0,1]$ can be taken as a measure of expectations. It can be assumed that each agent may have $j=1, \ldots K$ different expectations that define decisions on transactions $\boldsymbol{b s}_{i j}(t, x, y)(12 \mathrm{a})$ and each $j=1, \ldots K$ particular expectation has a particular measure in interval $[0,1]$.

To aggregate the value and importance of agents' expectations, the financial or economic value of a particular agent's expectation should be proportional to the value of transactions made under this expectation. Indeed, if particular transactions amount to $90 \%$ of all deals and are made under expectation 1 , then expectation 1 is ninety times more important than expectation 2 , which is responsible for only $1 \%$ of the same deals. Therefore, to aggregate the expectations of agents at point $x$, one should collect expectations weighted by the value of transactions made under these expectations.

Let us explain this statement using the transactions $b s_{i j}(t, z)(12 a)$ as example. We remind, that each transaction $b s_{i j}(t, z), z=(x, y)(12 a)$ defines the quantity $Q_{i j}$ and the cost $C_{i j}$ of the transaction. Decisions on the quantity $Q_{i j}$ and $\operatorname{cost} C_{i j}$ may be made under different expectations. The seller and 
the buyer may make decisions on the transaction between them under different expectations too. Therefore even single transaction $b s_{i j}(t, z)$ (12a) may be performed under four different expectations: Two expectations of the seller on the quantity $Q_{i j}$ and cost $C_{i j}$ and two expectations of the buyer. As example, we argue expectations that determine decisions of the sellers on quantity $Q_{i j}$ and $\operatorname{cost} C_{i j}$ of the transaction (12a). We denote the seller's expectations $e^{2} x_{Q i}(k ; t, x)$ of type $k=1, \ldots K$ of agent $i$ at point $x$ to perform the transactions of the amount $Q_{i j}(k ; t, z)$ as part of the transactions of the total amount $Q_{i j}(t, z)$ (12a) of agent $i$ with agent $j$ under all expectations. Let us denote the expectations $\operatorname{ex}_{C i}(l ; t, x)$ of type $l=1, \ldots K$ of the same agent to perform the transaction of amount $Q_{i j}(k ; t, z)$ at the cost- $C_{i j}(l ; t, z)$. It seems reasonable that the decisions on the quantity $Q_{i j}(k ; t, z)$ depend on the decisions on $\operatorname{cost} C_{i j}(l ; t, z)$ of transactions and vice versa. Therefore, the amount $Q_{i j}$ and the cost $C_{i j}$ of transactions preformed by the seller should depend on both expectations $k$ and $l: e x_{Q i}(k, l ; t, x)$ and $e x_{C i}(k, l ; t, x)$. We denote the volume $Q_{i j}$ and the $\operatorname{cost} C_{i j}$ of the seller's transaction $b s_{i j}(t, z)(12 \mathrm{a})$ as:

$$
\boldsymbol{b s}_{i j}(t, \boldsymbol{z})=\left(Q_{i j}(\boldsymbol{k} ; t, \boldsymbol{z}) ; C_{i j}(\boldsymbol{k} ; t, \boldsymbol{z})\right) ; \boldsymbol{k}=(k, l) ; k, l=1, \ldots K ; \boldsymbol{z}=(\boldsymbol{x}, \boldsymbol{y})
$$

The same reasons allow denote the volume $Q_{i j}$ and the $\operatorname{cost} C_{i j}$ of the buyer's transaction $b s_{i j}(t, z)$ (12a) as:

$$
\boldsymbol{b} s_{i j}(t, z)=\left(Q_{i j}(t, z ; l) ; C_{i j}(t, z ; l)\right) ; \boldsymbol{l}=(k, l) ; k, l=1, \ldots K
$$

To define the economic value of the sellers' expectations $e x_{Q i}(k, l ; t, x)$ and $e x_{C i}(k, l ; t, x)$ let us introduce the sellers' expected transaction $\boldsymbol{e t}_{i j}(\boldsymbol{k} ; t, \boldsymbol{x}, \boldsymbol{y})$ as follows:

$$
\begin{gathered}
e t_{i j}(\boldsymbol{k} ; t, \boldsymbol{z})=\left(e t_{Q i j}(\boldsymbol{k} ; t, \boldsymbol{z}) ; e t_{C i j}(\boldsymbol{k} ; t, \boldsymbol{z})\right) ; \boldsymbol{k}=(k, l) \\
e t_{Q i j}(\boldsymbol{k} ; t, \boldsymbol{z})=e x_{Q i}(\boldsymbol{k} ; t, \boldsymbol{x}) Q_{i j}(\boldsymbol{k} ; t, \boldsymbol{z}) ; e t_{C i j}(\boldsymbol{k} ; t, \boldsymbol{z})=e x_{C j}(\boldsymbol{k} ; t, \boldsymbol{y}) C_{i j}(\boldsymbol{k} ; t, \boldsymbol{z})
\end{gathered}
$$

The relations (19c) describe the sellers' volume expected transaction $e t_{Q i}(k ; t, z)$ that equals the product of the volume expectations $e x_{Q i}(k ; t, x)$ of type $k, k=(k, l)$ at point $x$ and the quantity $Q_{i j}(k, t, z)$ of the transaction performed between agents $i$ at $x$ as the sellers and agents $j$ at $y$ as the buyers. The cost of expected transactions $e t_{C i}(k ; t, z)(19 \mathrm{c})$ equals the product of the cost expectations $e_{C i}(k ; t, x)$ of type $l$, $k=(k, l)$ at a point $x$ and the $\operatorname{cost} C_{i j}(k ; t, z)$ of transaction performed between agents $i$ at $x$ as sellers and agents $j$ at $y$ as buyers. Similar considerations define buyers expected transactions:

$$
\begin{gathered}
\boldsymbol{e t}_{i j}(t, z ; l)=\left(\operatorname{et}_{Q i j}(t, z ; l) ; \operatorname{et}_{C i j}(t, z ; l)\right) ; \boldsymbol{l}=(k, l) \\
e t_{Q i j}(t, z ; l)=e x_{Q i}\left(t, x ; l_{1}\right) Q_{i j}(t, z ; l) ; e t_{C i j}(t, z ; l)=e x_{j}\left(t, y ; l_{2}\right) C_{i j}(t, z ; l)
\end{gathered}
$$

Now let us move from the description of transactions between agents to the description of transactions between points of the economic domain (12c) and (12d) similar to (12f)-(12j) and define a part $Q(k ; t, z)$ of the total amount $Q(t, z)$ and a cost $C(k ; t, z)$ of the total cost $C(t, z)$ of the transaction (12h) performed under sellers' expectations of the type $k$ :

$$
\begin{aligned}
& Q(\boldsymbol{k} ; t, \boldsymbol{z})=\sum_{i \in d V(\boldsymbol{x}) ; j \in d V(\boldsymbol{y}) ; \Delta} Q_{i, j}(\boldsymbol{k} ; t, \boldsymbol{z}) ; z=(\boldsymbol{x}, \boldsymbol{y}) \\
& C(\boldsymbol{k} ; t, \boldsymbol{z})=\sum_{i \in d V(\boldsymbol{x}) ; j \in d V(\boldsymbol{y}) ; \Delta} C_{i, j}(\boldsymbol{k} ; t, \boldsymbol{z}) ; \boldsymbol{k}=(k, l)
\end{aligned}
$$

The total amount $Q(t, z)(12 \mathrm{i})$ and the total $\operatorname{cost} C(t, z)$ of the transactions (12h) equal sum by all sellers' expectations and by all buyers' expectations:

$$
Q(t, z)=\sum_{k l} Q(k, l ; t, z)=\sum_{k l} Q(t, z ; k, l)
$$




$$
C(t, z)=\sum_{k l} C(k, l ; t, z)=\sum_{k l} C(t, z ; k, l)
$$

Now we define sellers' expected transactions $E t_{s}(k ; t, z), z=(x, y)$ between points $x$ and $y$ made under sellers' expectations $k=(k, l)$. Let us aggregate $(19 \mathrm{c})$ alike to $(15 \mathrm{~d})-(15 \mathrm{~g})$ as:

$$
\begin{aligned}
E t_{S}(\boldsymbol{k} ; t, \boldsymbol{z}) & =\left(E t_{Q}(\boldsymbol{k} ; t, \boldsymbol{z}) ; E t_{C}(\boldsymbol{k} ; t, \boldsymbol{z})\right) ; \boldsymbol{z}=(\boldsymbol{x}, \boldsymbol{y}) \\
E t_{Q}(\boldsymbol{k} ; t, \boldsymbol{z}) & =\sum_{i \in d V(\boldsymbol{x}) ; j \in d V(\boldsymbol{y}) ; \Delta} e x_{i}\left(k_{1} ; t, \boldsymbol{x}\right) Q_{i j}(\boldsymbol{k} ; t, \boldsymbol{z}) \\
E t_{C}(\boldsymbol{k} ; t, \boldsymbol{z}) & =\sum_{i \in d V(\boldsymbol{x}) ; j \in d V(\boldsymbol{y}) ; \Delta} e x_{j}\left(k_{2} ; t, \boldsymbol{y}\right) C_{i j}(\boldsymbol{k} ; t, \boldsymbol{z})
\end{aligned}
$$

The relations (20a)-(20c) and the functions $Q(k ; t, z)(19 \mathrm{e})$ and $C(k ; t, z)$ (19f) define sellers' expectations $E x_{Q}(k ; t, z)$ and $E x_{Q}(k ; t, z)$ of the type $k=(k, l)$ as:

$$
\begin{aligned}
& E t_{Q}(\boldsymbol{k} ; t, \boldsymbol{z})=E x_{Q}(\boldsymbol{k} ; t, \boldsymbol{z}) Q(\boldsymbol{k} ; t, \boldsymbol{z}) \\
& E t_{C}(\boldsymbol{k} ; t, \boldsymbol{z})=E x_{C}(\boldsymbol{k} ; t, \boldsymbol{z}) C(\boldsymbol{k} ; t, \boldsymbol{z})
\end{aligned}
$$

We underline, that sellers' expected transactions $E t_{Q}(\boldsymbol{k} ; t, z), E t_{C}(\boldsymbol{k} ; t, z)(20 \mathrm{~b})$ and (20c) and sellers' expectations $E x_{Q}(k ; t, z), E x_{C}(k ; t, z)(20 \mathrm{~d})$ and (20e) are determined with respect to the transactions (12f)-(12j) with the selected financial or economic variable $E$. The transactions with a different variables $E$-with commodities, service, assets, etc.-define different expectations $E x_{Q}(k ; t, z), E x_{C}(k ; t, z)$. To define macro expectations of sellers' $E x_{Q}(k ; t)$ and $E x_{C}(k ; t)$ at moment $t$ let us take the integrals over the economic domain $(12 \mathrm{c})$ and $(12 \mathrm{~d})$ :

$$
\begin{gathered}
Q(\boldsymbol{k} ; t, \boldsymbol{x})=\int d y Q(\boldsymbol{k} ; t, \boldsymbol{x} ; \boldsymbol{y}) ; C(\boldsymbol{k} ; t, \boldsymbol{x})=\int d \boldsymbol{y} C(\boldsymbol{k} ; t, \boldsymbol{x} ; \boldsymbol{y}) \\
E t_{Q}(\boldsymbol{k} ; t, \boldsymbol{x})=\int d \boldsymbol{y} E t(\boldsymbol{k} ; t, \boldsymbol{x}, \boldsymbol{y})=E x_{Q}(\boldsymbol{k} ; t, \boldsymbol{x}) Q(\boldsymbol{k} ; t, \boldsymbol{x}) \\
E t_{C}(\boldsymbol{k} ; t, \boldsymbol{x})=\int d \boldsymbol{y} E t(\boldsymbol{k} ; t, \boldsymbol{x}, \boldsymbol{y})=E x_{C}(\boldsymbol{k} ; t, \boldsymbol{x}) C(\boldsymbol{k} ; t, \boldsymbol{x}) \\
Q(\boldsymbol{k} ; t)=\int d x d y Q(\boldsymbol{k} ; t, \boldsymbol{x} ; \boldsymbol{y}) ; C(\boldsymbol{k} ; t)=\int d x d y C(\boldsymbol{k} ; t, \boldsymbol{x} ; \boldsymbol{y}) \\
E t_{Q}(\boldsymbol{k} ; t)=\int d x d \boldsymbol{y} E t(\boldsymbol{k} ; t, \boldsymbol{x}, \boldsymbol{y})=E x_{Q}(\boldsymbol{k} ; t) Q(\boldsymbol{k} ; t) \\
E t_{C}(\boldsymbol{k} ; t)=\int d x d \boldsymbol{y} E t(\boldsymbol{k} ; t, \boldsymbol{x}, \boldsymbol{y})=E x_{C}(\boldsymbol{k} ; t) C(\boldsymbol{k} ; t)
\end{gathered}
$$

The relations (21a) define the amount $Q(\boldsymbol{k} ; t, \boldsymbol{x})$ of the transactions (12f) with variable $E$ performed by sellers at $x$ under their expectations with all buyers of entire economics. The functions $C(k ; t, x)$ (21a) define the cost of sellers' transactions of the amount $Q(k ; t, x)(21 a)$ with all buyers. The relations (21b) define sellers expected transactions $E t_{Q}(k ; t, x)$ of the amount $Q(k ; t, x)$ under sellers' expectations $E x_{Q}(k ; t, x)$ with all buyers. The relations (20c) define sellers expected transactions $E t_{C}(k ; t, x)$ of the cost $C(\boldsymbol{k} ; t, \boldsymbol{x})$ under sellers' expectations $E x_{C}(\boldsymbol{k} ; t, x)$ with all buyers. The relations (21d) define the volume $Q(k ; t)$ of all transactions with variable $E$ performed in the economics under sellers' expectations $E x_{Q}(k ; t)$ (20e) of the type $k=(k, l)$. The relations (21d) define the $\operatorname{cost} C(k ; t)$ of all transactions with variable $E$ performed in the economics under sellers' expectations $E x_{C}(k ; t)(21 \mathrm{f})$. Therefore, starting with the definitions of the sellers' expected transactions (20a)-(20c) and the definitions of $Q(k ; t, z)(19 \mathrm{e})$ and $C(k ; t, z)$ (19f) we derive reasonable definitions of macro sellers expectations of the volume $E x_{Q}(k ; t)(21 \mathrm{e})$ and the cost $E x_{C}(k ; t)$ (21f) for transactions with variable $E$. Similar relations (21a)-(21f) are valid for the buyers' volume $Q(t, z ; l)$, cost $C(t, z ; l)$, and buyers' expectations $E x_{Q}(t ; l)$, and $E x_{C}(t ; l)$. We outline, that 
expectations of the type $k=(k, l)$ play different role for the transactions with a different variables $E$. That makes observations, measurements, and description of financial expectations a really complex problem.

Now we explain and describe how expected transactions and expectations can flow on the domain (12c-4) alike to flows of variables (6)-(10) and transactions (14a)-(15i). For brevity we take only flows of the amount $Q_{i j}(t, z)$ (12a) of sellers' transactions $\boldsymbol{b s}_{i j}(t, z)$ (12a). The flows of sellers' $\operatorname{cost} C_{i j}(t, z)(12 \mathrm{a})$ and the flows of buyers' expected transactions are determined in the same way. The motion of agents $i$ and $j$ at points $x$ and $y$ with the velocities $\boldsymbol{v}_{i}(t, x)$ and $\boldsymbol{v}_{j}(t, y)(14 \mathrm{c})$ and (14d) due to change of their risk ratings induces flows $p_{Q i j}(k ; t, z)$ of sellers' expected transactions et $t_{Q i j}(k ; t, z)(19 \mathrm{c})$ alike to flows $p_{Q i j x}(t, z)$ (14c) of the amount $Q_{i j}(t, z)$ (12a) of the transactions $b s_{i j}(t, z)$ (12a) as:

$$
\boldsymbol{p} e_{Q i j}(\boldsymbol{k} ; t, z)=e t_{Q i j}(\boldsymbol{k} ; t, z) \boldsymbol{v}_{i}(\boldsymbol{x})=e x_{i}(\boldsymbol{k} ; t, x) Q_{i, j}(\boldsymbol{k} ; t, \boldsymbol{z}) \boldsymbol{v}_{i}(x)
$$

The functions $p \boldsymbol{e}_{Q i j}(\boldsymbol{k} ; t, \boldsymbol{z})$ (22a) describe flows of expected transactions $e_{\mathrm{Q} i j}(\boldsymbol{k} ; t, \boldsymbol{z}), \boldsymbol{k}=(k, l)$ carried by agent $i$ with velocity $v_{i}$. To define the aggregate flows of sellers' expected transactions we collect flows $p e_{Q i j}(k ; t, z)$ of the expected transactions $e t_{Q i j}(k ; t, z)(22 \mathrm{a})$ of agents $i$ in a unit $d V(t, z)(12 \mathrm{e})$ and then average the sum during a time term $\Delta$ similar to (12f)-(12i) as:

$$
\boldsymbol{P e}_{Q x}(\boldsymbol{k} ; t, \boldsymbol{z})=\sum_{i \in d V(x) ; j \in d V(y) \Delta} e t_{Q i j}(\boldsymbol{k} ; t, \boldsymbol{z}) v_{i}(\boldsymbol{x})
$$

The sellers move along axis $X(12 \mathrm{c})$ and (12d) and we note the flow of sellers' expected transactions as $\boldsymbol{P} \boldsymbol{e}_{Q x}(\boldsymbol{k} ; t, \boldsymbol{z})$. Let us note the buyers flows as $\boldsymbol{P e _ { Q y }}(t, z ; l)$.

$$
\boldsymbol{P \boldsymbol { e } _ { Q x }}(\boldsymbol{k} ; t, \boldsymbol{z})=E t_{Q}(\boldsymbol{k} ; t, \boldsymbol{z}) \boldsymbol{v} \boldsymbol{e}_{Q x}(\boldsymbol{k} ; t, \boldsymbol{z})=E x_{Q}(\boldsymbol{k} ; t, \boldsymbol{z}) Q(\boldsymbol{k} ; t, \boldsymbol{z}) \boldsymbol{v} \boldsymbol{e}_{Q x}(\boldsymbol{k} ; t, \boldsymbol{z})
$$

The relations (22b) and (22c) define the aggregated flows $\boldsymbol{P} \boldsymbol{e}_{\mathrm{Q}}(\boldsymbol{k} ; t, z)$ and the velocities $\boldsymbol{v} \boldsymbol{e}_{\mathrm{Q} x}(\boldsymbol{k} ; t, \boldsymbol{z})$ of sellers expected transactions $E x_{Q}(k ; t, z)$ of the type $k=(k, l)$. Similar to definitions of the flows of variables (6)-(9) and the flows of transactions (14a)-(15i), the integrals of (22b) and (22c) by $d z=d x d y$ over domain (12c) and (12d) define the macro flows $\boldsymbol{P} \boldsymbol{e}_{\mathrm{Q} x}(\boldsymbol{k} ; t)$ and the macro velocities $\boldsymbol{v} \boldsymbol{e}_{\mathrm{Q}}(\boldsymbol{k} ; \boldsymbol{t})$ of expected transactions $E t_{Q x}(k ; t)$ and expectations $E x_{Q x}(k ; t)(21 \mathrm{e})$ as:

$$
\begin{aligned}
& \boldsymbol{P} \boldsymbol{e}_{Q x}(\boldsymbol{k} ; t)=\int d z \boldsymbol{P} \boldsymbol{e}_{\mathrm{Q} x}(\boldsymbol{k} ; t, \boldsymbol{z}) \\
& \boldsymbol{P \boldsymbol { e } _ { Q x }}(\boldsymbol{k} ; t)=E t_{Q x}(\boldsymbol{k} ; t) \boldsymbol{v} \boldsymbol{e}_{Q x}(\boldsymbol{k} ; t)=E x_{Q x}(\boldsymbol{k} ; t) Q(\boldsymbol{k} ; t) \boldsymbol{v} \boldsymbol{e}_{Q x}(\boldsymbol{k} ; t)
\end{aligned}
$$

The relations (22d) and (22e) define the sellers' macro flows of $\boldsymbol{P} \boldsymbol{e}_{Q x}(\boldsymbol{k} ; t)$ and the velocities $\boldsymbol{v} \boldsymbol{e}_{Q x}(\boldsymbol{k} ; t)$ of expected transaction $E t_{Q x}(\boldsymbol{k} ; t)$ and describe the motion of expectations $E x_{Q x}(\boldsymbol{k} ; t)$ of variable $E$. Borders of the domain (12c) and (12d) reduce the motion along risk axes and hence values and direction of flows $\boldsymbol{P} \boldsymbol{e}_{Q x}(\boldsymbol{k} ; t)$ and velocities $\boldsymbol{v} \boldsymbol{e}_{Q x}(\boldsymbol{k} ; t)$ should fluctuate. These induce time oscillations of expectations $E x_{Q x}(k ; t)$. Such fluctuations of expectations $E x_{Q x}(k ; t)$ should correlate with fluctuations of the volume $Q(k ; t)(21 \mathrm{~d})$ and $Q(t)(13 \mathrm{~g})$ of transactions induced by oscillations of the flows $\boldsymbol{P}(t)(16 \mathrm{~b})$ and velocities $\boldsymbol{v}(t)(16 \mathrm{~d})$. We propose that fluctuations of macro variables, transactions, and expectations induced by oscillations of their flows and velocities due to borders of the economic domain (12c) and (12d) should be treated as business cycles. We present simple business cycles models in Olkhov (2017c, 2019).

We underline, that velocities of $\boldsymbol{v}_{x}(t)$ of the sellers and velocities $\boldsymbol{v}_{y}(t)$ of the buyers (15i) differ from velocities $\boldsymbol{v} \boldsymbol{e}_{\mathrm{Qx}}(\boldsymbol{k} ; t)$ of the sellers' expectations $E x_{\mathrm{Qx}}(\boldsymbol{k} ; t)$ and velocities $\boldsymbol{v} \boldsymbol{e}_{\mathrm{Q} y}(t ; \boldsymbol{l})$ of the buyers' expectations $E x_{Q y}(t ; l)$. The flows and velocities of expectations for a different $k=(k, l)$ are also different. The flows of a different variables $E$, transactions, and expectations have different velocities and their mutual interactions on the economic domain (12c) and (12d) reflect extreme complexity of real financial and economic processes. 
The definitions of sellers expected transactions $E t_{Q x}(k ; t, z)(20 \mathrm{~b})$, their flows $P e_{Q x}(k ; t, z)$ and velocities $v_{Q x}(k ; t, z)(22 b)$ and (22c), allow take equations on expected transactions and their flows similar to equations on transactions and their flows $(17 \mathrm{~d}),(17 \mathrm{e})$ as:

$$
\begin{aligned}
& \frac{\partial}{\partial t} E t_{Q x}(\boldsymbol{k} ; t, \boldsymbol{z})+\nabla \cdot\left(E t_{Q x}(\boldsymbol{k} ; t, \boldsymbol{z}) \boldsymbol{v} \boldsymbol{e}_{Q x}(\boldsymbol{k} ; t, \boldsymbol{z})\right)=W_{Q x}(\boldsymbol{k} ; t, \boldsymbol{z}) \\
& \frac{\partial}{\partial t} \boldsymbol{P} \boldsymbol{e}_{Q x}(\boldsymbol{k} ; t, \boldsymbol{z})+\nabla \cdot\left(\boldsymbol{P} \boldsymbol{e}_{Q x}(\boldsymbol{k} ; t, \boldsymbol{z}) \boldsymbol{v} \boldsymbol{e}_{Q x}(\boldsymbol{k} ; t, \boldsymbol{z})\right)=\boldsymbol{R}_{Q x}(\boldsymbol{k} ; t, \boldsymbol{z})
\end{aligned}
$$

The functions $W_{Q x}, W_{Q y}$ and $\boldsymbol{R}_{Q x}, \boldsymbol{R}_{Q y}$ in Equations (22f) and (22g) describe the action of financial variables, transactions, and different expectations, and other factors that may impact changes of expected transactions $E t_{Q x}(\boldsymbol{k} ; t, z)$ and their flows $\boldsymbol{P} \boldsymbol{e}_{Q x}(\boldsymbol{k} ; t, z)$. Equations on sellers' and buyers' expected transactions that determine the cost $C(t, z)(12 \mathrm{j})$ of the transactions (12h) are similar to (22f), (22g). Similar to (18a) and (18b) equations on $E t_{Q x}(\boldsymbol{k} ; t)(21 \mathrm{e})$ and flows $\boldsymbol{P} \boldsymbol{e}_{\mathrm{Qx}}(\boldsymbol{k} ; t)(22 \mathrm{~d})$ take form:

$$
\begin{aligned}
& \frac{d}{d t} E t_{Q x}(\boldsymbol{k} ; t)=W_{Q x}(\boldsymbol{k} ; t)=\int d z W_{Q x}(\boldsymbol{k} ; t, \boldsymbol{z}) \\
& \frac{d}{d t} \boldsymbol{P e}_{Q x}(\boldsymbol{k} ; t)=\boldsymbol{R}_{Q x}(\boldsymbol{k} ; t)=\int d z \boldsymbol{R}_{Q x}(\boldsymbol{k} ; t, \boldsymbol{z})
\end{aligned}
$$

The Equations (11b) and (11c) on financial variables $A(t, x)$ and their flows $\boldsymbol{P}_{A}(t, \boldsymbol{x})$, Equations $(17 \mathrm{~d})$ and (17e) on the volume $Q(t, z)(12 \mathrm{i})$ of the transactions $B S(t, z)(12 \mathrm{~h})$, and transactions' flows $\boldsymbol{P}_{Q}(t, z)$ (15b) and (15c), and Equations (22f), (22g) on expected transaction $E t_{Q x}(\boldsymbol{k} ; t, z)$, and flows $\boldsymbol{P} \boldsymbol{e}_{Q x}(\boldsymbol{k} ; t, \boldsymbol{z})$ complete our approximation of the macro financial system based on a description of relations between variables, transactions, and expectations in the domain (12c) and (12d). It is obvious, that description of any particular problem requires the definition of the right-hand side factors of the Equations (11b) and (12c), (17d) and (17e), (22f) and (22g). All specifics and details of the financial processes are hidden in and are determined by the functions $F_{A}(t, x)$ and $G_{A}(t, x), F(t, z)$ and $G(t, z), W_{Q x}, W_{Q y}$ and $\boldsymbol{R}_{Q x}$, $\boldsymbol{R}_{Q y}$. We apply our methods and equations to describe the wave propagation of small disturbances of variables (Olkhov 2016a, 2016b, 2017a), the wave propagation of disturbances of transactions (Olkhov 2018a) and the surface waves (Olkhov 2017b). Our methods permit model the business cycles (Olkhov 2017c, 2019), describe hidden complexities of the classical Black-Scholes option pricing model (Olkhov 2016a, 2016b) and propose Lorentz attractor as a possible model origin of the random behavior of price fluctuations (Olkhov 2018b). In the next section we describe how perturbations of transactions may define price and return disturbances.

\section{Asset Pricing and Return}

Asset pricing is one of the most important problems of macro finance. We refer (Cochrane and Hansen 1992; Cochrane and Culp 2003; Hansen 2013; Campbell 2014; Fama 2014; Cochrane 2017) as only small part of asset pricing studies. In this section we describe how economic equations on variables, transactions, expectations, and their flows can govern asset prices, returns, and their fluctuations.

The Equations (17d) and (17e) describe the volume $Q(t, z)(12 \mathrm{i})$ of the transaction $B S(t, z)(12 \mathrm{~h})$ with economic variable $E$ and similar equations model the cost $C(t, z)(12 \mathrm{j})$ of the transaction $B S(t, z)$. As variable $E$ one may study any particular assets and Equations (17d), (17e) define relations on price and price fluctuations. We remind, that different aggregations of the volume and cost of the transactions define different prices. For example, the relations $(12 \mathrm{k})$ define the price of transactions with the volume $Q(t, z)$ and cost $C(t, z)$ between points $x$ and $y, z=(x, y)$ and (13c) define the price $p_{S}(t, x)$ of the sellers of variable $E$ from point $x$. The transactions of the quantity $Q(k ; t, z)(19 \mathrm{e})$ with the cost 
$C(\boldsymbol{k} ; t, z)$ (19f) performed under sellers' expectations of the type $k=(k, l)$ determine the price $p(\boldsymbol{k} ; t, z)$ of sellers' transactions:

$$
C(\boldsymbol{k} ; t, \boldsymbol{z})=p(\boldsymbol{k} ; t, \boldsymbol{z}) Q(\boldsymbol{k} ; t, \boldsymbol{z}) ; \boldsymbol{k}=(k, l)
$$

The relations (12f) define the price $p(t)$ of all transactions with the volume $Q(t)$ (12f) with $\operatorname{cost} C(t)$ (12f) with selected assets $E$ performed in the economy at a moment $t$. The price $p(k ; t)$ of all transactions with the volume $Q(k ; t)$ with $\operatorname{cost} C(\boldsymbol{k} ; t)(21 \mathrm{~d})$ with selected assets $E$ performed under expectations $\boldsymbol{k}=$ $(k, l)$ in the economy at a moment $t$.

$$
C(\boldsymbol{k} ; t)=p(\boldsymbol{k} ; t) Q(\boldsymbol{k} ; t) ; \boldsymbol{k}=(k, l)
$$

The relations (12k), (13c), (12f), (23a), and (23b) indicate that the price of assets always should be treated in regard to definite aggregation of the volume and the cost of transactions. Equations on transactions and their flows define equations on prices. As the simplest case we study The Equations (18a) and (18b) on the volume $Q(t)$ and the flow of volume $\boldsymbol{P}_{Q}(t)$ of transactions

$$
\frac{d}{d t} Q(t)=F_{Q}(t) ; \frac{d}{d t} P_{Q}(t)=G_{Q}(t)
$$

and similar equations on the $\operatorname{cost} C(t)$ and the flow of volume $\boldsymbol{P}_{C}(t)$ of transactions

$$
\frac{d}{d t} C(t)=F_{C}(t) ; \frac{d}{d t} \boldsymbol{P}_{C}(t)=\boldsymbol{G}_{C}(t)
$$

The Equations (23c) and (23d) define equations on the price $p(t)(12 \mathrm{f})$ of all transactions made in the economy at a moment $t$ with variable $E$

$$
\begin{gathered}
\frac{d}{d t} Q(t)=F_{Q}(t) ; Q(t) \frac{d}{d t} p(t)+p(t) F_{Q}(t)=F_{C}(t) \\
Q(t) \frac{d}{d t} v_{Q}(t)+F_{Q}(t) \boldsymbol{v}_{Q}(t)=G_{Q}(t) ; Q(t) p(t) \frac{d}{d t} \boldsymbol{v}_{C}(t)+\boldsymbol{v}_{C}(t) F_{C}(t)=G_{C}(t) \\
\boldsymbol{P}_{Q}(t)=Q(t) \boldsymbol{v}_{Q}(t) ; \boldsymbol{P}_{C}(t)=C(t) \boldsymbol{v}_{C}(t)=Q(t) p(t) \boldsymbol{v}_{C}(t)
\end{gathered}
$$

Even the simplest form of the price Equations (23e)-(23g) demonstrates that the right hand factors hide the main complexity of the price dynamics and price may depend on the flows $\boldsymbol{P}_{Q}(t), \boldsymbol{P}_{C}(t)$ or velocities $\boldsymbol{v}_{Q}(t), \boldsymbol{v}_{C}(t)$ of amount $Q(t)$ and cost $C(t)$ of transactions.

Currently we do not know any research of possible impact of the risk flows $\boldsymbol{P}_{Q}(t), \boldsymbol{P}_{C}(t)$ and velocities $\boldsymbol{v}_{Q}(t), \boldsymbol{v}_{C}(t)$ on the price $p(t)$ evolution and fluctuations. These studies may be very important for description of price and return disturbances and for modeling the price-volume and return-volume relations.

For simplicity here we neglect possible impact of flows and describe how equations on volume and cost similar to (23e) may model the price fluctuations. Let us study equations similar to (23e)-(23g) that take into account the volume $Q(k ; t)$ and the $\operatorname{cost} C(k ; t)(21 \mathrm{~d})$ that define the price $p(k ; t)(23 \mathrm{~b})$ for different expectations $k=(k, l)$. Similar to $(23 \mathrm{e})-(23 \mathrm{~g})$ equations on $Q(k ; t)$ and $C(k ; t)(24 \mathrm{a})$ take form:

$$
\frac{d}{d t} Q(\boldsymbol{k} ; t)=F_{Q}(\boldsymbol{k} ; t) ; \frac{d}{d t} C(\boldsymbol{k} ; t)=F_{C}(\boldsymbol{k} ; t)
$$

Let us assume, that $F_{Q}(k ; t)$ and $F_{C}(k ; t)$ in (24a) depend on the expected transaction $E t_{Q}(k ; t)(21 \mathrm{e})$ and $E t_{C}(k ; t)(21 \mathrm{f})$ that due to (22f) and (18a) follows equations similar to (24a)

$$
\frac{d}{d t} E t_{Q}(\boldsymbol{k} ; t)=F e_{Q}(\boldsymbol{k} ; t) ; \frac{d}{d t} E t_{C}(\boldsymbol{k} ; t)=F e_{C}(\boldsymbol{k} ; t)
$$


Let us assume, that $F e_{Q}(\boldsymbol{k} ; t)$ and $F e_{C}(\boldsymbol{k} ; t)$ in $(24 \mathrm{~b})$ depend on the volume $Q(\boldsymbol{k} ; t)$ and the cost $C(\boldsymbol{k} ; t)$ (24a). Let us take small dimensionless perturbations of $Q(\boldsymbol{k} ; t), C(\boldsymbol{k} ; t), E t_{Q}(\boldsymbol{k} ; t)$, and $E t_{C}(\boldsymbol{k} ; t)$ as:

$$
\begin{gathered}
Q(\boldsymbol{k} ; t)=Q_{0 k l}(1+q(\boldsymbol{k} ; t)) ; C(\boldsymbol{k} ; t)=C_{0 k l}(1+c(\boldsymbol{k} ; t)) \\
\boldsymbol{k}=(k, l) ; k, l=1, \ldots K \\
E t_{Q}(\boldsymbol{k} ; t)=E t_{Q 0 k l}\left(1+e t_{q}(\boldsymbol{k} ; t)\right) ; E t_{C}(\boldsymbol{k} ; t)=E t_{C 0 k l}\left(1+e t_{c}(\boldsymbol{k} ; t)\right)
\end{gathered}
$$

and assume that the mean values of $Q_{0 k l}, C_{0 k l}, E t_{Q 0 k l}, E t_{C O k l}$ are slow to compare with variations of the small disturbances $q(\boldsymbol{k} ; t), c(\boldsymbol{k} ; t), e t_{q}(\boldsymbol{k} ; t)$ and $e t_{c}(\boldsymbol{k} ; t)$. Let us take the same assumptions on the functions in the right-hand side of (24a) and (24b).

$$
\begin{gathered}
F_{Q}(\boldsymbol{k} ; t)=F_{Q 0 k l}\left(1+f_{Q}(\boldsymbol{k} ; t)\right) ; F_{C}(\boldsymbol{k} ; t)=F_{C O k l}\left(1+f_{\mathcal{c}}(\boldsymbol{k} ; t)\right) \\
F e_{Q}(\boldsymbol{k} ; t)=F e_{Q 0 k l}\left(1+f e_{Q}(\boldsymbol{k} ; t)\right) ; F e_{C l}(\boldsymbol{k} ; t)=F e_{C 0 k l}\left(1+f e_{c}(\boldsymbol{k} ; t)\right)
\end{gathered}
$$

Therefore, the mean values of $Q_{0 k l}, C_{0 k l}, E t_{Q 0 k l}, E t_{C 0 k l}$, and $F_{Q 0 k l}, F_{C 0 k l}, E t_{Q 0 k l}, E t_{C O k l}$ may be treated as constants and the equations on disturbances take form:

$$
\begin{gathered}
Q_{0 k l} \frac{d}{d t} q(\boldsymbol{k} ; t)=F_{\mathrm{Q} 0 k l} f_{q}(\boldsymbol{k} ; t) ; C_{0 k l} \frac{d}{d t} c(\boldsymbol{k} ; t)=F_{\mathrm{C} O k l} f_{c}(\boldsymbol{k} ; t) \\
E t_{\mathrm{Q} 0 k l} \frac{d}{d t} e t_{q}(\boldsymbol{k} ; t)=F e_{\mathrm{Q} 0 k l} f e_{q}(\boldsymbol{k} ; t) ; E t_{\mathrm{C} 0 k l} \frac{d}{d t} e t_{c}(\boldsymbol{k} ; t)=F e_{C 0 k l} f e_{c}(\boldsymbol{k} ; t)
\end{gathered}
$$

To consider (25a) and (25b) as self-consistent equations, assume that the disturbances $f_{q}(\boldsymbol{k} ; t)$ and $f_{c}(\boldsymbol{k} ; t)$ in $(25 \mathrm{a})$ depend on the disturbances $e t_{q}(\boldsymbol{k} ; t)$ and $e t_{c}(\boldsymbol{k} ; t)$ and $f e_{q}(\boldsymbol{k} ; t)$ and $f e_{c}(\boldsymbol{k} ; t)$ in $(25 \mathrm{~b})$ depend on the disturbances of $q(\boldsymbol{k} ; t)$ and $c(\boldsymbol{k} ; t)$.

$$
\begin{gathered}
f_{q}(\boldsymbol{k} ; t)=a_{q k l} l t_{q}(\boldsymbol{k} ; t) ; f_{c}(\boldsymbol{k} ; t)=a_{c k l} l t_{c}(\boldsymbol{k} ; t) \\
f e_{q}(\boldsymbol{k} ; t)=b e_{q k l} q(\boldsymbol{k} ; t) ; f e_{c}(\boldsymbol{k} ; t)=b e_{c k l} c(\boldsymbol{k} ; t)
\end{gathered}
$$

Due to (26a) and (26b) the Equations (25a) and (25b) take form:

$$
\begin{gathered}
Q_{0 k l} \frac{d}{d t} q(\boldsymbol{k} ; t)=a_{q k l} F_{\mathrm{Q} 0 k l} e t_{q}(\boldsymbol{k} ; t) ; C_{0 k l} \frac{d}{d t} c(\boldsymbol{k} ; t)=a_{c k l} F_{\mathrm{C} 0 k l} e t_{c}(\boldsymbol{k} ; t) \\
E t_{\mathrm{Q} 0 k l} \frac{d}{d t} e t_{q}(\boldsymbol{k} ; t)=b e_{q k l} F e_{\mathrm{Q} O k l} q(\boldsymbol{k} ; t) ; E t_{\mathrm{C} 0 k l} \frac{d}{d t} e t_{c}(\boldsymbol{k} ; t)=b e_{c k l} F e_{C 0 k l} c(\boldsymbol{k} ; t)
\end{gathered}
$$

For

$$
\omega_{q k l}^{2}=-a_{q k l} b e_{q k l} \frac{F_{\mathrm{Q} 0 k l}}{Q_{0 k l}} \frac{F e_{\mathrm{Q} 0 k l}}{E t_{\mathrm{Q} k l}}>0 ; \omega_{c k l}^{2}=-a_{c k l} b e_{c k l} \frac{F_{\mathrm{C} 0 k l}}{C_{0 k l}} \frac{F e_{\mathrm{C} 0 k l}}{E t_{\mathrm{C} 0 k l}}>0
$$

The equations on disturbances $q(\boldsymbol{k} ; t), c(\boldsymbol{k} ; t), e t_{q}(\boldsymbol{k} ; t), e t_{c}(\boldsymbol{k} ; t)$ take form of harmonic oscillators:

$$
\begin{gathered}
\left(\frac{d^{2}}{d t^{2}}+\omega_{q k l}^{2}\right) q(\boldsymbol{k} ; t)=0 ;\left(\frac{d^{2}}{d t^{2}}+\omega_{c k l}^{2}\right) c(\boldsymbol{k} ; t)=0 \\
k=(k, l) ; k, l=1, \ldots K \\
\left(\frac{d^{2}}{d t^{2}}+\omega_{q k l}^{2}\right) e t_{q}(\boldsymbol{k} ; t)=0 ;\left(\frac{d^{2}}{d t^{2}}+\omega_{c k l}^{2}\right) e t_{c}(\boldsymbol{k} ; t)=0
\end{gathered}
$$


Simple solutions of (27b) for the dimensionless disturbances $q(\boldsymbol{k} ; t)$ and $c(\boldsymbol{k} ; t)$

$$
\begin{aligned}
& q(\boldsymbol{k} ; t)=g_{q k l} \sin \omega_{q k l} t+d_{q k l} \cos \omega_{q k l} t g_{q k l}, d_{q k l} \ll 1 \\
& c(\boldsymbol{k} ; t)=g_{c k l} \sin \omega_{c k l} t+d_{c k l} \cos \omega_{c k l} t ; g_{c k l}, d_{c k l} \ll 1
\end{aligned}
$$

The Equations (27b) and (27c) describe simple harmonic fluctuations (27d) and (27e) of disturbances $q(\boldsymbol{k} ; t)$ of the volume $Q(\boldsymbol{k} ; t)$ and disturbance $c(\boldsymbol{k} ; t)$ of the $\operatorname{cost} C(\boldsymbol{k} ; t)$ of transactions under the expectations $k=(k, l)$. The harmonic Equations (27b), (27c) are result of the assumptions of linear dependence in (26a)-(26d) and describe the simplest possible model relations between the disturbances of $q(k ; t)$ of volume $Q(k ; t)$ and the disturbance $c(k ; t)$ of $\operatorname{cost} C(k ; t)$ of transactions on one hand and disturbances of expectations $e t_{q}(k ; t)$ and $e t_{c}(k ; t)$ on other hand. The disturbances of the cost $C(t)$ and the volume $Q(t)$ (12f) take form:

$$
\begin{aligned}
& Q(t)=\sum_{k, l} Q_{0 k l}(1+q(\boldsymbol{k} ; t))=Q_{0} \sum_{k, l} \lambda_{k l}(1+q(\boldsymbol{k} ; t)) \\
& C(t)=\sum_{k, l} C_{0 k l}(1+c(\boldsymbol{k} ; t))=C_{0} \sum_{k, l} \mu_{k l}(1+c(\boldsymbol{k} ; t))
\end{aligned}
$$

The relations (28a) describe impact of dimensionless disturbances $q(k ; t)$ on the volume $Q(t)$ and (28b) describe impact of dimensionless disturbances $c(k ; t)$ on the cost $C(t), k=(k, l)$ :

$$
\begin{gathered}
Q_{0}=\sum_{k, l} Q_{0 k l} ; \lambda_{k l}=\frac{Q_{0 k l}}{Q_{0}} ; C_{0}=\sum_{k, l} C_{0 k l} ; \mu_{k l}=\frac{C_{0 k l}}{C_{0}} \\
\sum \lambda_{k l}=\sum \mu_{k l}=1 ;
\end{gathered}
$$

The relations (12f) define the price $p(t)$ for transactions with the volume $Q(t)$ and cost $C(t)$ :

$$
p(t)=\frac{C(t)}{Q(t)}=\frac{\sum_{k, l} C(k, l ; t)}{\sum_{k, l} Q(k, l ; t)} ; p_{0}=\frac{C_{0}}{Q_{0}}=\frac{\sum_{k, l} C_{0 k l}}{\sum_{k, l} Q_{0 k l}}
$$

In linear approximation by the disturbances $q(k, l ; t)$ and $c(k, l ; t)$, the price $p(t)(28 \mathrm{e})$ take form:

$$
\begin{gathered}
p(t)=\frac{C(t)}{Q(t)}=\frac{C_{0} \sum_{k, l} \mu_{k l}(1+c(k, l ; t))}{Q_{0} \sum_{k, l} \lambda_{k l}(1+q(k, l ; t))}=p_{0}\left[1+\sum_{k, l} \mu_{k l} c(k, l ; t)-\sum_{k, l} \lambda_{k l} q(k, l ; t)\right] \\
p(t)=p_{0}[1+\pi(t)]=p_{0}\left[1+\sum_{k, l}\left(\mu_{k l} c(k, l ; t)-\lambda_{k l} q(k, l ; t)\right)\right]
\end{gathered}
$$

The dimensionless fluctuations of the price $\pi(t)$ (28f) equal the weighted sum of the disturbances $q(k, l ; t)$ and $c(k, l ; t)$ as $(28 \mathrm{~g})$ :

$$
\pi(t)=\sum_{k, l} \mu_{k l} c(k, l ; t)-\lambda_{k l} q(k, l ; t)
$$

Now, for (23b) and (24c) we present $\pi(t)$ in other form:

$$
C(k, l ; t)=C_{0 k l}[1+c(k, l ; t)]=p_{0 k l}[1+\pi(k, l ; t)] Q_{0 k l}[1+q(k, l ; t)]
$$

From (28f), (28g) and (29a) in the linear approximation by $c(k, l ; t), \pi(k, l ; t)$ and $q(k, l ; t)$ obtain:

$$
C_{0 k l}=p_{0 k l} Q_{0 k l} ; c(k, l ; t)=\pi(k, l ; t)+q(k, l ; t)
$$

Let us substitute $(29 \mathrm{~b})$ into $(28 \mathrm{~g})$ :

$$
\pi(t)=\sum_{k, l} \mu_{k l} \pi(k, l ; t)+\sum_{k, l}\left(\mu_{k l}-\lambda_{k l}\right) q(k, l ; t)
$$

The relations (29c) describe the price perturbations $\pi(t)$ as the weighted sum of partial price disturbances $\pi(k, l ; t)$ and volume disturbances $q(k, l ; t)$. Therefore, the statistics of price disturbances 
$\pi(t)$ should be defined by the statistics of partial price disturbances $\pi(k, l ; t)$ and the statistics of volume disturbances $q_{k}(k, l ; t)$. There are many studies of price-volume correlations (Campbell et al. 1993; Leung et al. 2002; Podobnik et al. 2009; DeFusco et al. 2017) but our simple model presents the relations (29c) that allow study the price-volume correlations under different approximations on statistics.

Relations between disturbances of the quantity and cost of transactions on one hand and disturbances of the expectations that approve these transactions may be the source of the random evolution. Random behavior of disturbances of the quantity and cost of the transactions induce random motion of the price disturbances $\pi(t)$ and in (Olkhov 2018b) we describe Lorentz attractor as a possible model that cause random behavior of the price fluctuations.

Return perturbations. The price disturbances $(29 \mathrm{c})$ cause perturbations of the return $r(t, d)$ :

$$
r(t, d)=\frac{p(t)}{p(t-d)}-1
$$

Let us introduce the partial returns $r(\boldsymbol{k} ; t, d)$ for the price $p(\boldsymbol{k} ; t)(23 \mathrm{~b})$ and the "returns" $w(k ; t, d)$ for the volumes $Q(k ; t)(24 \mathrm{c}), \boldsymbol{k}=(k, l)$ as:

$$
r(\boldsymbol{k} ; t, d)=r(k, l ; t, d)=\frac{p(k, l ; t)}{p(k, l ; t-d)}-1 ; w(k ; t, d)=w(k, l ; t, d)=\frac{Q(k, l ; t)}{Q(k, l ; t-d)}-1
$$

For simplicity assume, that the mean price $p_{0 k l}$ and $Q_{0 k l}(29 \mathrm{~b})$ are constant during a time term $d$ and present (30a) and (30b) as

$$
\begin{gathered}
r(t, d)=\frac{\pi(t)-\pi(t-d)}{1+\pi(t-d)} ; w(k, l ; t, d)=\frac{q(k, l ; t)-q(k, l ; t-d)}{1+q(k, l ; t-d)} \\
r(t, d)=\sum \mu_{k l} \frac{1+\pi(k, l ; t-d)}{1+\pi(t-d)} r(k, l ; t, d)+\sum\left(\mu_{k l}-\lambda_{k l}\right) \frac{1+q(k, l ; t-d)}{1+\pi(t-d)} w(k, l ; t, d)
\end{gathered}
$$

Let us define

$$
\begin{gathered}
\varepsilon_{k l}(t-d)=\mu_{k l} \frac{1+\pi(k, l ; t-d)}{1+\pi(t-d)} ; \eta_{k l}(t-d)=\left(\mu_{k l}-\lambda_{k l}\right) \frac{1+q(k, l ; t-d)}{1+\pi(t-d)} \\
\sum_{k, l}\left[\varepsilon_{k l}(t-d)+\eta_{k l}(t-d)\right]=1 \\
r(t, d)=\sum_{k, l} \varepsilon_{k l}(t-d) r(k, l ; t, d)+\sum_{k, l} \eta_{k l}(t-d) w(k, l ; t, d)
\end{gathered}
$$

The relations (30f) and (30g) describe the return (30a) as a sum of partial returns and the volume "returns" $w(k, l ; t, d)(30 \mathrm{~b})$ and (30c). The sum for coefficients $\mu_{k l}$ and $\left(\mu_{k l}-\lambda_{k l}\right)$ for the price $p(t)(28 \mathrm{f})$, $\pi(t)(29 \mathrm{c})$, and $\varepsilon_{k l}(t)$, and $\eta_{k l}(t)$ for the return $r(t, d)$ (30a) equals unit but (29c) and (30g) can't be treated as averaging procedure because some coefficients $\left(\mu_{k l}-\lambda_{k l}\right)$ and $\eta_{k l}(t)$ should be negative. If the mean price $(29 \mathrm{~b}) p_{0 k l}=p_{0}$ for all pairs of the expectations $(k, l)$ then from $(28 \mathrm{f})$ and $(28 \mathrm{~g})$ obtain

$$
p_{0 k l}=p_{0}=\text { const } \rightarrow \lambda_{k l}=\mu_{k l} ; \eta_{k l}(t)=0 \text { for all } k, l
$$

and the relations $(29 \mathrm{c})$ and $(30 \mathrm{~g})$ take simple form

$$
\begin{gathered}
\pi(t)=\sum_{k, l} \mu_{k l} \pi(k, l ; t) \\
r(t, d)=\sum_{k, l} \mu_{k l} \frac{1+\pi(k, l ; t-d)}{1+\pi(t-d)} r(k, l ; t, d)=\sum_{k, l} \mu_{k l} \frac{\pi(k, l ; t)-\pi(k, l ; t-d)}{1+\pi(t-d)}
\end{gathered}
$$

Therefore, the assumption (30h) on the prices $(29 \mathrm{~b})$ for all pairs of the expectations $(k, l)$ causes representation (30i) and (30j) of the price disturbances $\pi(t)$ as a sum of partial price disturbances $\pi(k, l ; t)$ 
weighted by $\mu_{k l}(28 \mathrm{c})$ for different pairs of expectations $(k, l)$. If the coefficients $\mu_{k l}(28 \mathrm{c})$ are random then their statistics should impact the statistic properties of the price disturbances $\pi(t)$. If (30h) is not valid then the price disturbances $\pi(t)$ should take form (29c) and depend on partial disturbances $\pi(k, l ; t)$, volume perturbations $q(k, l ; t)$, and statistics of $\lambda_{k l}$ and $\mu_{k l}$ (28c). Assumption (30h) cause returns as (30j), otherwise returns take (30g). Actually, expectations are the key factors for market competition and different expectations $(k, l)$ should cause different mean partial prices $p_{0 k l}$. That produces complex representation of the price $(29 \mathrm{c})$ and return $(30 \mathrm{~g})$ disturbances as well as impact volatility and statistic distributions of price and return disturbances.

\section{Conclusions}

Properties of macro finance and financial markets are constantly changing. Methods for their description should match these variations. We propose methods for macro modeling that may describe the different evolution of macro finance and financial markets. Above, we only presented the theoretical framework.

Let us resume with the main issues of our methods. We model macro finance and financial markets by three elements: Financial variables, market transactions, and expectations of economic agents. To do that, we distribute agents by their risk ratings as coordinates in the economic domain and describe variables, transactions, and expectations as density functions of risk. We regard risks as the main drivers of any economic or financial evolution and consider the financial activity of agents as the main source of risk. Any financial or economic activity is related to risk.

We show that changes of agents' risk ratings due to any reason induce financial or economic flows of variables, transactions, and expectations in the economic domain and these flows impact the evolution of macro finance and financial markets. Flows of variables, market transactions, and expectations at least double the number of properties that define the evolution of financial markets. Different variables as demand and supply have different flows and their mutual interactions cause permanent perturbations of any market supply and demand equilibrium state. That makes the existence of market supply-demand equilibrium and equilibrium between different markets an exceptional case.

Financial variables as functions of risk $x$ describe the state of the macro system. Market transactions as functions of risk $z=(x, y)$ describe the dynamics of financial variables and model the evolution of financial markets. Expectations as most "etheric" substances impact agents to perform transactions. Numerous expectations are determined by variables, transactions, expectations of other agents, technology or climate forecasts, etc. These factors project the impact of the economic environment on financial processes and can be considered as the main source of macro modeling complexity. Different expectations cause different impacts on market transactions. The economic or financial value of expectations, as well as their importance and influence on market evolution, should be measured proportionally to the amount of market transactions performed under these expectations. That makes the description of financial markets an exciting problem.

We have already used our model to describe several interesting properties of macro financial systems. Modeling financial variables and market transactions in economic space uncovers the existence of numerous financial waves. Wave generation, propagation, and interactions reflect most general and common properties of any complex system. A description of economic and financial waves requires space where these waves can propagate. Modeling in economic space uncovers various types of economic and financial waves that may impact macro financial evolution and sustainability. In (Olkhov 2016a, 2016b, 2017a, 2018a) we describe the wave propagation of disturbances of financial variables and market transactions in the economic domain for different models of interactions between them. Perturbations of financial variables or market transactions near borders of the economic domain may generate waves that are similar to surface waves in fluids (Olkhov 2017b). Financial relations that cause wave propagation are completely different from physical waves. These distinctions may allow the exponential growth of small disturbances of wave amplitudes in time and that have an important impact on macro finance. Partitions of collective financial variables and market transactions 
in the economic domain permit mean values to be defined for the risk coordinate of each variable or transaction. Borders of the economic domain (1), (12c) and (12d) reduce the change of these mean risk coordinates. This causes a cyclical motion of mean risks that reflects the business and credit cycles of macro financial systems (Olkhov 2017c, 2019). Mutual interactions between price, volume, and expectation perturbations may become stochastic due to models similar to Lorentz attractors (Olkhov 2018b). These examples indicate that the methods we propose may model various properties of financial systems.

In this paper, we have applied our methods to describe price and return perturbations. We derived simple relations to show that the statistical properties of asset pricing and return disturbances should be studied with respect to the corresponding volume perturbation of the market transactions. Price-volume correlations have been under investigation for decades, (Campbell et al. 1993; Leung et al. 2002; Podobnik et al. 2009; DeFusco et al. 2017) but our model provides simple representation for description correlations under different assumptions and a tool for modeling their statistical properties. It is obvious that our simple model may not match the real properties of measured price-volume and return-volume correlations. Additionally, it redirects the studies of price, return, and volume statistics to the investigation of relations between market transactions and expectations.

The applicability of our methodology does not require any specific assumptions. A description of financial markets as the most practical problem should be based on econometric data. The use of our model is based on the assumption that econometrics may provide sufficient data for risk rating assessments of economic agents. This requires the development of risk assessment methods that may model risk ratings in the economic domain (1) for continuous risk grades and Big Data analysis and computing. A description of financial markets in the economic domain requires the modeling of numerous equations on financial variables, transactions, and expectations, as well as their flows and mutual interactions that have certain parallels to problems of multi-fluid hydrodynamics. Solutions to these problems are impossible without modern computing. We propose that the implementation of our methods may be useful for financial authorities, central banks, top commercial banks and corporations, and top investment and hedge funds. We are sure that our methods can improve the description and forecasting of financial processes.

Funding: This research received no external funding, assistance, specific grant or financial support from TVEL or funding agencies in the public, commercial, or not-for-profit sectors.

Conflicts of Interest: The authors declare no conflict of interest.

\section{References}

Ackerman, Frank. 1999. Still Dead After All These Years: Interpreting the Failure of General Equilibrium Theory. Medford: Global Development and Environment Institute, Tufts University.

Altman, Edward, Herbert Rijken, Dan Balan, Matthew Watt, Juan Forero, and Mina Jorge, eds. 2010. The Z-MetricsTM Methodology for Estimating Company Credit Ratings and Default Risk Probabilities. New York: RiskMetrics Group, Inc.

Arrow, Kenneth J. 1974. General Economic Equilibrium: Purpose, Analytic Techniques, Collective Choice. The American Economic Review 64: 253-72.

Arrow, Kenneth J., and Gerard Debreu. 1954. Existence of an Equilibrium for a Competitive Economy. Econometrica 22: 265-90. [CrossRef]

Bangia, Anil, Francis X. Diebold, André Kronimus, Christian Schagen, and Til Schuermann. 2000. Ratings Migration and the Business Cycle, With Applications to Credit Portfolio Stress Testing. Penn Institute for Economic Research Working Paper No. 01-004. Philadelphia: Univ. of Pennsylvania.

Blume, Lawrence E., and David Easley. 1984. Rational Expectations Equilibrium: An Alternative Approach. Journal of Economic Theory 34: 116-29. [CrossRef]

Borland, Lisa, and Jean-Philipp Bouchaud. 2005. On a multi-timescale statistical feedback model for volatility fluctuations. arXiv, arXiv:physics/0507073. 
Brock, William A., and Cars H. Hommes. 1998. Heterogeneous beliefs and routes to chaos in a simple asset pricing model. Journal of Economic Dynamics and Control 22: 1235-74. [CrossRef]

Brunnermeier, Markus K., and Jonathan A. Parker. 2005. Optimal Expectations. American Economic Review 95: 1092-118. [CrossRef]

Campbell, John Y. 2014. Empirical Asset Pricing: Eugene Fama, Lars Peter Hansen, and Robert Shiller. Scandinavian Journal of Economics 116: 593-634. [CrossRef]

Campbell, John Y., Sanford J. Grossman, and Jiang Wang. 1993. Trading Volume and Serial Correlation in Stock Returns. The Quarterly Journal of Economics 108: 905-39. [CrossRef]

Chakraborti, Anirban, Ioane Muni Toke, Marco Patriarca, and Frédéric Abergel. 2011. Econophysics review: II. Agent-based models. Quantitative Finance 11: 1013-41. [CrossRef]

Cochrane, John H. 2017. Macro-Finance. Review of Finance 21: 945-85. [CrossRef]

Cochrane, John H., and Christopher L. Culp. 2003. Equilibrium Asset Pricing and Discount Factors: Overview and Implications for Derivatives Valuation and Risk Management. In Modern Risk Management: A History. London: Incisive RWG Ltd., pp. 57-92.

Cochrane, John H., and Lars P. Hansen. 1992. Asset Pricing Explorations for Macroeconomics. NBER Macroeconomics Annual 7: 115-82. [CrossRef]

DeFusco, Anthony A., Charles G. Nathanson, and Eric Zwick. 2017. Speculative Dynamics of Prices and Volume. Cambridge: National Bureau of Economic Research (NBER).

Del Negro, Marco, Stefano Eusepi, Marc Giannoni, Argia Sbordone, Andrea Tambalotti, Matthew Cocci, Raiden Hasegawa, and Henry Linder. 2013. The FRBNY DSGE Model. Staff Reports No. 647. New York: Federal Reserve Bank of New York.

Dhesi, Gurjeet, Bilal Shakeel, and Marcel Ausloos. 2019. Modelling and forecasting the kurtosis and returns distributions of financial markets: Irrational fractional Brownian motion model approach. Annals of Operations Research, 1-14. [CrossRef]

Dominitz, Jeff, and Charles F. Manski. 2005. Measuring and Interpreting Expectations of Equity Returns. Cambridge: National Bureau of Economic Research (NBER).

Fama, Eugene F. 2014. Two Pillars of Asset Pricing. American Economic Review 104: 1467-85. [CrossRef]

Farmer, Roger E. A. 2017. Post Keynesian Dynamic Stochastic General Equilibrium Theory. Chicago and Cambridge: National Bureau of Economic Research (NBER).

Fernández-Villaverde, Jesús. 2010. The econometrics of DSGE models. Journal of the Spanish Economic Association 1: 3-49. [CrossRef]

Fitch Ratings, Inc. 2017. Asia-Pacific Structured Finance 2016 Transition and Default Study. New York: Fitch Ratings, Inc., pp. 1-14.

Fitch Ratings, Inc. 2018. Procedures and Methodologies for Determining Credit Ratings. New York: Fitch Ratings, Inc.

Gabaix, Xavier, Parameswaran Gopikrishnan, Vasiliki Plerou, and H. Eugene Stanley. 2003. A theory of power-law distributions in financial market fluctuations. Nature 423: 267-70. [CrossRef] [PubMed]

Gontis, Vigyntas, Shlomo Havlin, Aleksejus Kononovicius, Boris Podobnik, and H. Eugene Stanley. 2016. Stochastic model of financial markets reproducing scaling and memory in volatility return intervals. Physica A 462: 1091-102. [CrossRef]

Greenwood, Robin, and Andrei Shleifer. 2014. Expectations of Returns and Expected Returns. The Review of Financial Studies 27: 714-46. [CrossRef]

Guedesa, Everaldo F., Paulo Ferreira, Andreia Dionisiod, and Gilney F. Zebendee. 2018. An econophysics approach to study the effect of BREXIT referendum on European Union stock markets. Physica A 523: 1175-82. [CrossRef]

Haldane, Andrew G., and Arthur E. Turrell. 2018. An interdisciplinary model for macroeconomics. Oxford Review of Economic Policy 34: 219-51. [CrossRef]

Hansen, Lars P. 2013. Uncertainty Outside and Inside Economic Models. Chicago: Nobel Prize Lecture, University of Chicago.

Hansen, Lars P., and Thomas J. Sargent. 1979. Formulating and Estimating Dynamic Rational Expectations Models. Cambridge: NBER.

Hazlitt, Henry. 1959. The Failure of the "New Economics": An Analysis of The Keynesian Fallacies. Princeton: D. Van Nostrand Company, Inc. 
Janžek, Tine, and Petra Ziherl. 2013. Overview of Models and Methods for Measuring Economic Agent's Expectations. IFC Bulletin 36. Basel: BIS, pp. 172-79.

Keynes, John M. 1936. The General Theory of Employment, Interest and Money. London: Macmillan Cambridge University Press.

Klaauw, Wilbert, Wändi B. de Bruin, Giorgio Topa, Simon Potter, and Michael Bryan. 2008. Rethinking the Measurement of Household Inflation Expectations: Preliminary Findings; New York: Federal Reserve Board (FRB).

Komunjer, Ivana, and Serena Ng. 2011. Dynamic Identification of Dynamic Stochastic General Equilibrium Models. Econometrica 79: 1995-2032.

Kydland, Finn, and Edward C. Prescott. 1980. A Competitive Theory of Fluctuations and the Feasibility and Desirability of Stabilization Policy. In Rational Expectations and Economic Policy. Edited by Stanley Fisher. Cambridge: National Bureau of Economic Research (NBER), pp. 169-98.

Kydland, Finn, and Edward C. Prescott. 1990. The Econometrics of the General Equilibrium Approach to Business Cycles; Staff Report 130. Minneapolis: Federal Reserve Board (FRB).

Leung, Charles, Garion Lau, and Youngman Leong. 2002. Testing Alternative Theories of the Property Price-Trading Volume Correlation. Journal of Real Estate Research 23: 253-63.

Lof, Matthijs. 2014. Essays on Expectations and the Econometrics of Asset Pricing. MPRA. Available online: http://mpra.ub.uni-muenchen.de/59064/ (accessed on 3 April 2019).

Lucas, Robert E. 1972. Expectations and the Neutrality of Money. Journal of Economic Theory 4: 103-24. [CrossRef]

Manski, Charles. 2004. Measuring Expectations. Econometrica 72: 1329-76. [CrossRef]

Manski, Charles. 2017. Survey Measurement of Probabilistic Macroeconomic Expectations: Progress and Promise. Cambridge: National Bureau of Economic Research (NBER).

Mantegna, Rosario N., and H. Eugene Stanley. 2000. An Introduction to Econophysics: Correlations and Complexity in Finance. Cambridge: Cambridge University Press, pp. 1-147.

McNeil, Alexander J., Rüdiger Frey, and Paul Embrechts. 2005. Quantitative Risk Management. Concepts, Techniques and Tools. Princeton: Princeton University Press.

Metz, Albert, and Richard Cantor. 2007. Introducing Moody's Credit Transition Model. New York: Moody's Investor Service, pp. 1-26.

Moody's Investors Service. 2010. Rating Symbols and Definitions. New York: Moody's Investors Service.

Moody's Investors Service. 2018. Procedures and Methodologies Used to Determine Credit Ratings. New York: Moody's Investors Service.

Morgenstern, Oskar. 1972. Thirteen Critical Points in Contemporary Economic Theory: An Interpretation. Journal of Economic Literature 10: 1163-89.

Muth, John F. 1961. Rational Expectations and the Theory of Price Movements. Econometrica 29: 315-35. [CrossRef] Olkhov, Victor. 2016a. On Economic space Notion. International Review of Financial Analysis 47: 372-81. [CrossRef]

Olkhov, Victor. 2016b. Finance, Risk and Economic space. ACRN Oxford Journal of Finance and Risk Perspectives 5: 209-21.

Olkhov, Victor. 2017a. Quantitative Wave Model of Macro-Finance. International Review of Financial Analysis 50: 143-50. [CrossRef]

Olkhov, Victor. 2017b. Credit-Loans Non-Local Transactions and Surface-Like Waves. Available online: https://ssrn.com/abstract=2971417 (accessed on 14 June 2019).

Olkhov, Victor. 2017c. Econophysics of Business Cycles: Aggregate Economic Fluctuations, Mean Risks and Mean Square Risks. arXiv, arXiv:1709.00282.

Olkhov, Victor. 2018a. How Macro Transactions Describe the Evolution and Fluctuation of Financial Variables. International Journal of Financial Studies 6: 38. [CrossRef]

Olkhov, Victor. 2018b. Expectations, Price Fluctuations and Lorenz Attractor. MPRA. Available online: https://mpra.ub.uni-muenchen.de/89105/ (accessed on 7 May 2019).

Olkhov, Victor. 2019. Economic Transactions Govern Business Cycles. ACRN Oxford Journal of Finance and Risk Perspectives 7: 102-22. [CrossRef]

Podobnik, Boris, Davor Horvaticd, Alexander M. Petersena, and H. Eugene Stanley. 2009. Cross-correlations between volume change and price change. Proceedings of the National Academy of Sciences of the USA (PNAS) 106: 22079-84. [CrossRef]

Poggio, Tomaso, Andrew W. Lo, Blake LeBaron, and Nicholas T. Chan. 1999. Agent-Based Models of Financial Markets: A Comparison with Experimental Markets. Cambridge: MIT Sloan School of Management. 
Roehner, Bertrand M. 2010. Fifteen Years of Econophysics: Worries, Hopes and Prospects. Science and Culture 76: 305-14.

S\&P. 2014. Guide to Credit Rating Essentials. What Are Credit Ratings and How do They Work? New York: McGraw Hill Financial.

S\&P. 2016. SEP Global Ratings Definitions. New York: Standard \& Poor's, pp. 1-45.

S\&P. 2019. 2018 Annual Global Corporate Default and Rating Transition Study. New York: Standard \& Poor's, pp. 1-148.

Sargent, Thomas J., and Neil Wallace. 1976. Rational Expectations And The Theory Of Economic Policy. Journal of Monetary Economics 2: 169-83. [CrossRef]

Schinckus, Christophe. 2012. Methodological comment on Econophysics review I and II: Statistical econophysics and agent-based Econophysics. Quantitative Finance 12: 1189-92. [CrossRef]

South, Andrew H., and Zev R. Gurwitz. 2018. 2017 Annual Global Structured Finance Default Study and Rating Transitions. New York: S\&P Global Ratings.

Stangl, Anna. 2009. Essays on the Measurement of Economic Expectations. Inaugural dissertation, Ludwig-Maximilians-University, Munich, Germany.

Stanley, Eugene. 2003. Statistical Physics and Financial Fluctuations: Do Outlet Exist? Physica A 318: $279-92$. [CrossRef]

Starr, Ross M. 2011. General Equilibrium Theory, An Introduction. New York: Cambridge University Press.

Stiglitz, Joseph E. 2017. Where Modern Macroeconomics Went Wrong. Cambridge: National Bureau of Economic Research (NBER).

Strauss, Walter A. 2008. Partial Differential Equations. An Introduction. Hoboken: John Wiley \& Sons, p. 179.

Tanaka, Mari, Nicholas Bloom, Joel M. David, and Maiko Koga. 2018. Firm Performance and Macro Forecast Accuracy. Cambridge: National Bureau of Economic Research (NBER).

Tesfatsion, Leigh, and Kenneth Judd. 2005. Handbook of Computational Economics: Agent-Based Computational Economics. Amsterdam: Elsevier, vol. 2.

Thaler, Richard H. 2018. From Cashews to Nudges: The Evolution of Behavioral Economics. American Economic Review 108: 1265-87. [CrossRef]

Vines, David, and Samuel Wills, eds. 2018a. Rebuilding macroeconomic theory. Oxford Review of Economic Policy 34: 2-353.

Vines, David, and Samuel Wills. 2018b. The rebuilding macroeconomic theory project: An analytical assessment. Oxford Review of Economic Policy 34: 1-42. [CrossRef] 